Research Article

\title{
Two-Dimensional ISAR Fusion Imaging of Block Structure Targets
}

\author{
Xiaoxiu Zhu $\mathbb{D}^{D}$, Limin Liu $\left(\mathbb{D}\right.$, Baofeng Guo $\mathbb{D}^{\circ}$, Wenhua Hu ${ }^{(D)}$, Lin Shi, and Juntao Ma \\ Department of Electronic and Optical Engineering, Army Engineering University Shijiazhuang Campus, \\ Shijiazhuang 50003, China \\ Correspondence should be addressed to Baofeng Guo; 15132497492@126.com and Wenhua Hu; hwhsaq@sina.com
}

Received 11 April 2021; Revised 8 July 2021; Accepted 22 July 2021; Published 2 August 2021

Academic Editor: Francisco Falcone

Copyright (c) 2021 Xiaoxiu Zhu et al. This is an open access article distributed under the Creative Commons Attribution License, which permits unrestricted use, distribution, and reproduction in any medium, provided the original work is properly cited.

The range resolution and azimuth resolution are restricted by the limited transmitting bandwidth and observation angle in a monostatic radar system. To improve the two-dimensional resolution of inverse synthetic aperture radar (ISAR) imaging, a fast linearized Bregman iteration for unconstrained block sparsity (FLBIUB) algorithm is proposed to achieve multiradar ISAR fusion imaging of block structure targets. First, the ISAR imaging echo data of block structure targets is established based on the geometrical theory of the diffraction model. The multiradar ISAR fusion imaging is transformed into a signal sparse representation problem by vectorization operation. Then, considering the block sparsity of the echo data of block structure targets, the FLBIUB algorithm is utilized to achieve the block sparse signal reconstruction and obtain the fusion image. The algorithm further accelerates the iterative convergence speed and improves the imaging efficiency by combining the weighted back-adding residual and condition number optimization of the basis matrix. Finally, simulation experiments show that the proposed method can effectively achieve block sparse signal reconstruction and two-dimensional multiradar ISAR fusion imaging of block structure targets.

\section{Introduction}

High-resolution inverse synthetic aperture radar (ISAR) images can provide information such as the size and geometric structure of targets, which is conducive to target recognition and classification [1-4]. In a monostatic ISAR imaging system, the range resolution and azimuth resolution are restricted by the limited transmitting bandwidth and observation angle, respectively $[5,6]$. The multiradar fusion imaging technology is utilized to fuse multiangle and multiband echo data measured by multiple radars with different frequency bands at different observation angles to improve the two-dimensional resolution of images [7].

Compressive sensing (CS) theory [8] can accurately reconstruct sparse signals by using a small number of measurements, which breaks the Nyquist sampling theorem and greatly reduces the sampling number. Since the echo data in ISAR imaging has a sparse property, the CS theory can be applied to ISAR fusion imaging [9]. The multiradar observation echo data can be regarded as the sampling data, and sparse reconstruction algorithms can be utilized to achieve two-dimensional ISAR fusion imaging based on sparse representation.

The targets usually have some block structure characteristics in practice scenes, such as satellites and aircraft with complex structures [10]. The nonzero scattering coefficients of block structure targets are considered continuously located in the imaging scene. It is necessary to consider the block structure characteristics and the correlation of the block sparse echo signal in ISAR imaging of block structure targets. Block sparse reconstruction algorithms are utilized to achieve high-resolution two-dimensional ISAR fusion imaging of block structure targets. Block sparse reconstruction algorithms mainly include greedy iterative algorithms, convex optimization algorithms, and Bayesian framework-based algorithms. Greedy iterative algorithms represented by the block orthogonal matching pursuit (BOMP) algorithm [11] are easy to implement and have low 
computational complexity. However, these algorithms cannot guarantee convergence to the global optimum, which affects the reconstruction accuracy. Convex optimization algorithms utilize the $l_{2,1}$ norm instead of the $l_{2,0}$ norm in block sparse signal reconstruction. The $l_{2} / l_{1}$-optimization program (L-OPT) can be regarded as a secondorder cone optimization program (SOCP), which can be solved by standard software packages [12]. The reconstruction accuracy of convex optimization algorithms is better than that of greedy iterative algorithms; however, the computational time is long and not suitable for large-scale signal reconstruction. Block sparse Bayesian learning (BSBL) algorithm [13] and pattern coupled-sparse Bayesian learning (PC-SBL) algorithm [14] are typical Bayesian framework-based algorithms, which automatically estimate parameters by Bayesian inference. The Bayesian framework-based algorithms can accurately reconstruct the sparse signals. However, a large number of matrix inversion operations are involved in the Bayesian inference and parameter estimation, which is also not suitable for largescale sparse signal reconstruction.

Due to the two-dimensional coupling of the fusion imaging signal, the linear system model of the two-dimensional multiradar ISAR fusion imaging based on sparse representation is established by signal vectorization. Large-scale signal reconstruction problems are involved. A simple, fast, and effective block sparse reconstruction algorithm should be utilized to achieve ISAR fusion imaging of block structure targets. Yin et al. [15] proposed that the Bregman iterative algorithms can quickly and effectively solve convex optimization problems and are applied to solve CS problems successfully. Linearized Bregman iteration (LBI) algorithm and fast linearized Bregman iteration (FLBI) algorithm are proposed in $[16,17]$, which can further improve the iterative convergence speed. Li et al. [18,19] applied the LBI-based algorithms into ISAR imaging, which have fast imaging capability and antinoise performance. However, the algorithms are only utilized to achieve ISAR imaging for point scattering targets, which are not suitable for the imaging of block structure targets.

A fast linearized Bregman iteration for unconstrained block sparsity (FLBIUB) algorithm is proposed to achieve two-dimensional multiradar ISAR fusion imaging of block structure targets. The multiradar fusion imaging model is established based on sparse representation. Considering the block sparsity characteristics of the echo data of block structure targets, the sparse representation problem is transformed into an unconstrained block sparsity optimization problem, which can be solved by an LBI-based algorithm. Moreover, the weighted back-adding residual and condition number optimization of the basis matrix are utilized to further accelerate the iterative convergence speed and improve the imaging efficiency. Simulation experiments verify the effectiveness and superiority of the proposed algorithm.

The rest of the paper is organized as follows. Section 2 gives the two-dimensional multiradar ISAR fusion imaging model based on sparse representation. Section 3 proposes the FLBIUB algorithm and summarizes the implementation process. Section 4 presents and discusses the performance of the proposed algorithm via both simulation and real data experiments. Conclusions are drawn in Section 5.

\section{Two-Dimensional Multiradar ISAR Fusion Imaging Model}

Multiradar ISAR fusion imaging technology exploits echo data obtained by multiple radars working in different frequency bands and different observation angles to fuse into a larger bandwidth and larger observation angle. It is a new approach to improve the two-dimensional resolution remarkably. Since the scattering coefficients of the scatterers are varying with frequency under wide bandwidth and small-angle observation conditions, the traditional ideal scatterer model is not suitable to characterize the scattering characteristics. Considering the variation of scattering coefficients with frequency, the ISAR imaging echo data is established based on the geometrical theory of diffraction (GTD) model [20].

2.1. ISAR Imaging Model. The echo data of the target can be described as the sum of the electromagnetic scattering of multiple independent scatterers in the high-frequency area. Suppose that ISAR transmits a chirp signal, the target has $P$ independent scatterers. After motion compensation, the echo data in the range frequency-azimuth slow time domain based on the GTD model can be expressed as

$$
S_{f}\left(f, t_{n}\right)=\sum_{p=1}^{P} \sigma_{p}\left(j \frac{f}{f_{0}}\right)^{\alpha_{p}} \cdot \exp \left[-j \frac{4 \pi}{c} f \Delta R_{p}\left(t_{n}\right)\right]
$$

where $f$ is the frequency, $t_{n}=\mathrm{nT}_{r}$ is the slow time, $n=0,1, \ldots, N-1, N$ is the pulse number, $T_{r}$ is the pulse repetition time, $f_{0}$ is the start frequency, and $c$ is the speed of electromagnetic waves. $\Delta R_{p}\left(t_{n}\right)=y_{p} \cos \left(\Delta \theta\left(t_{n}\right)\right)+x_{p}$ $\sin \left(\Delta \theta\left(t_{n}\right)\right)$ is the instantaneous distance between the $p-t h$ scatterer and the reference point, where $\left(x_{p}, y_{p}\right)$ is the coordinate of the $p$-th scatterer and ${ }_{1} \Delta \theta\left(t_{n}\right)$ is the cumulative observation angle within $t_{n} . \sigma_{p}$ and $\alpha_{p}$ are the constant scattering coefficient and the frequency-dependent factor (FDF) of the $p$ - th scatterer, respectively. The typical scatterer types and corresponding FDF values are shown in Table 1.

The imaging model can be approximated as a turntable model after motion compensation. Under the small-angle observation condition, we have the approximation as $\cos \left(\Delta \theta\left(t_{n}\right)\right) \approx 1$ and $\sin \left(\Delta \theta\left(t_{n}\right)\right) \approx \Delta \theta\left(t_{n}\right)$. Equation (1) can be approximated as

$$
S_{f}\left(f, t_{n}\right) \approx \sum_{p=1}^{P} \sigma_{p}\left(j \frac{f}{f_{0}}\right)^{\alpha_{p}} \cdot \exp \left[-j \frac{4 \pi}{c} f\left(y_{p}+x_{p} \Delta \theta\left(t_{n}\right)\right)\right] .
$$

Assuming that the angular rotation velocity of the uniform rotation of the turntable model is $\omega$, the cumulative observation angle can be expressed as $\Delta \theta\left(t_{n}\right)=$ 
TABLE 1: The typical types of scatterers and corresponding FDF values.

\begin{tabular}{lc}
\hline Scatterer type & FDF value \\
\hline Flat surface reflection & 1 \\
Single curved surface reflection & 0.5 \\
Point scattering & 0 \\
Edge diffraction & -0.5 \\
Corner diffraction & -1 \\
\hline
\end{tabular}

$\omega t_{n}=n \omega T_{r}(n=0,1, \ldots, N-1)$. Let $f=f_{0}+m \Delta f$ $(m=0,1, \ldots, M-1)$, where $\Delta f$ is the frequency sampling interval. $M$ and $N$ are the frequency sampling number and the angle sampling number, respectively. After the migration through resolution cells correction, the echo data in equation (2) can be discretized as

$$
\begin{aligned}
S(m, n)= & \sum_{p=1}^{P} \sigma_{p}\left(j \frac{f_{0}+m \Delta f}{f_{0}}\right)^{\alpha_{p}} \cdot \exp \left(-j \frac{4 \pi}{c} \Delta f y_{p} m\right) \\
& \cdot \exp \left(-j \frac{4 \pi}{c} f_{0} x_{p} \omega T_{r} n\right) .
\end{aligned}
$$

Let $\quad 2 \Delta f y_{p} / c=k / K(k=0,1, \ldots, K-1) \quad$ and $2 f_{0} x_{p} \omega T_{r} / c=l / L(l=0,1, \ldots, L-1)$, where $K \geq M$ and $L \geq N$. Referring to $[21,22]$, the imaging scene can be discretized into a two-dimensional grid with the size of $L \times K$. Since the scattering coefficients vary with frequency, the amplitude and phase of the echo data are coupled. The coupled two-dimensional echo data need to be vectorized to establish the imaging model based on sparse representation. Equation (3) can be vectorized as

$$
\mathbf{s}=\widetilde{\Psi} \widetilde{a},
$$

where $\mathbf{s}$ is the echo data vector with the size of $\mathrm{MN} \times 1$, which can be expressed as

$$
\begin{aligned}
s= & {[S(0,0), \ldots, S(M-1,0), S(0,1), \ldots, S(M-1,1), \ldots,} \\
& S(0, N-1), \ldots, S(M-1, N-1)]^{T},
\end{aligned}
$$

$\widetilde{\Psi}$ is the basis matrix with the size of $\mathrm{MN} \times 5 \mathrm{KL}$, which can be expressed as

$$
\widetilde{\Psi}=\left[\begin{array}{lllll}
\widetilde{\Omega}_{-1} & \widetilde{\Omega}_{-0.5} & \widetilde{\Omega}_{0} & \widetilde{\Omega}_{0.5} & \widetilde{\Omega}_{1}
\end{array}\right]_{\mathrm{MN} \times 5 \mathrm{KL}},
$$

where $\widetilde{\Omega}_{i}=\Gamma_{i} \mathbf{W} \quad(i=-1,-0.5,0,0.5,1)$, and $i$ corresponds to the FDF value. $\Gamma_{i}$ is the block diagonal matrix with the size of $\mathrm{MN} \times \mathrm{MN}$, which can be expressed as

$$
\Gamma_{i}=I_{N \times N} \otimes \mathrm{T}_{i}=\left[\begin{array}{ccc}
\mathrm{T}_{i} & \cdots & 0 \\
\vdots & \ddots & \vdots \\
0 & \cdots & \mathrm{T}_{i}
\end{array}\right]_{\mathrm{MN} \times \mathrm{MN}},
$$

where $\mathbf{I}$ is the identity matrix, $\otimes$ represents the Kronecker product, $\mathrm{T}_{i}$ is denoted as

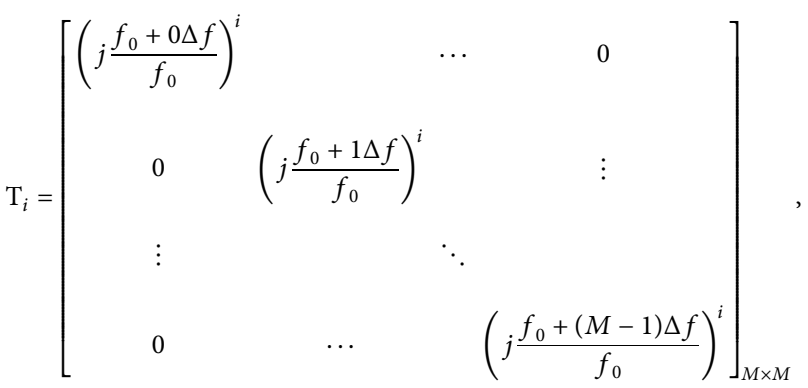

and $\mathbf{W}$ is a two-dimensional coupled dictionary matrix with the size of $\mathrm{MN} \times \mathrm{KL}$, which can be expressed as

$$
\mathbf{W}=\left[\begin{array}{llllll}
\mathbf{W}_{0} & \mathbf{W}_{1} & \cdots & \mathbf{W}_{n} & \cdots & \mathbf{W}_{N-1}
\end{array}\right]^{T},
$$

where

$$
\begin{aligned}
\mathbf{W}_{n}= & {[\Phi(0,0), \Phi(1,0), \ldots, \Phi(K-1,0), \Phi(0,1), \ldots,} \\
& \Phi(K-1, L-1)]_{M \times K L},
\end{aligned}
$$

$$
\Phi(k, l)=\left[\begin{array}{c}
\exp \left(-j 2 \pi\left(\frac{k \cdot 0}{K}+\frac{l n}{L}\right)\right) \\
\exp \left(-j 2 \pi\left(\frac{k \cdot 1}{K}+\frac{l n}{L}\right)\right) \\
\vdots \\
\exp \left(-j 2 \pi\left(\frac{k \cdot(M-1)}{K}+\frac{l n}{L}\right)\right)
\end{array}\right]_{M \times 1}
$$

$\tilde{a}=\left[\tilde{a}_{-1}{ }^{T}, \tilde{a}_{-0.5}{ }^{T}, \widetilde{a}_{0}{ }^{T}, \tilde{a}_{0.5}{ }^{T}, \tilde{a}_{1}^{T}\right]^{T}$ is the scattering coefficient vector with the size of $5 \mathrm{KL} \times 1$, where $\tilde{a}_{i}(i=-1,-0,5,0,0.5,1)$ is the vector of the ISAR image which is corresponding to the FDF values.

2.2. Multiradar ISAR Fusion Imaging Model. We take two radars with different frequency bands and different observation angles as an example to achieve the multiradar ISAR fusion imaging. The echo data of the independent radars have been preprocessed by motion compensation and mutual-coherence compensation. The scattering information received by the two radars cannot be too different to ensure achieving fusion imaging.

The frequency of radar 1 is denoted as $f_{0} \ldots f_{M_{1}-1}$ with $M_{1}$ frequency sampling points. The frequency of radar 2 is denoted as $f_{M-M_{2}} \ldots f_{M-1}$ with $M_{2}$ frequency sampling points. Radar 1 has $N_{1}$ observation angles as $\theta_{0} \ldots \theta_{N_{1}-1}$. Radar 2 has $N_{2}$ observation angles as $\theta_{N-N_{2}} \ldots \theta_{N-1} . \Delta f$ and $\Delta \theta$ are denoted as the frequency sampling interval and the angle sampling interval, respectively. $M$ and $N$ are denoted as the frequency sampling number and the angle sampling number of the full-band and full-angle echo data, respectively. The frequency band and the observation angle of the full-band and full-angle echo data can be expressed as $f_{m}=$ $f_{0}+m \Delta f(m=0,1, \ldots, M-1) \quad$ and $\theta_{n}=\theta_{0}+n \Delta \theta$ $(n=0,1, \ldots, N-1)$, respectively. The observation model of 
the two radars for ISAR fusion imaging is shown in Figure 1, where the red grids and the blue grids represent the observation data of radar 1 and radar 2, respectively. The blank grids are the echo data corresponding to the missing frequency band and observation angle.

Denote the vector $\bar{s}$ as the observation echo data of radar 1 and radar 2. $\bar{s}$ can be regarded as intercepted from the fullband and full-angle radar echo data s. The vectorized twodimensional multiradar ISAR fusion imaging model based on sparse representation can be expressed as

$$
\bar{s}=\mathbf{D s}=\mathbf{D} \widetilde{\Psi} \widetilde{a}=\widetilde{F} \widetilde{a},
$$

where $\widetilde{F}=\mathbf{D} \widetilde{\Psi}$ is the basis matrix corresponding to the observation echo data, and $\mathbf{D}$ is the measurement matrix which is denoted as

$$
\mathbf{D}=\left[\begin{array}{cc}
\mathbf{I}_{N_{1} \times N_{1}} \otimes \boldsymbol{\Psi}_{1} & 0_{M_{1} N_{1} \times\left(N-N_{1}\right) M} \\
0_{M_{2} N_{2} \times\left(N-N_{2}\right) M} & \mathbf{I}_{N_{2} \times N_{2}} \otimes \Psi_{2}
\end{array}\right]_{\left(M_{1} N_{1}+M_{2} N_{2}\right) \times M N},
$$

$$
\begin{aligned}
& \boldsymbol{\Psi}_{1}=\left[\begin{array}{ll}
\mathbf{I}_{M_{1} \times M_{1}} & \left.0_{M_{1} \times\left(M-M_{1}\right.}\right)
\end{array}\right]_{M_{1} \times M}, \\
& \boldsymbol{\Psi}_{2}=\left[\begin{array}{ll}
0_{M_{2} \times\left(M-M_{2}\right)} & \mathbf{I}_{M_{2} \times M_{2}}
\end{array}\right]_{M_{2} \times M},
\end{aligned}
$$

where $\mathbf{I}$ and 0 are the identity matrix and the zero matrix, respectively.

Since the echo data satisfies the spatial sparsity in ISAR imaging of block structure targets, block sparse reconstruction algorithms can be utilized to solve equation (12). The transmitting bandwidth and the observation angle are equivalently improved via ISAR fusion imaging technology, thereby simultaneously improving the two-dimensional resolution of ISAR imaging.

The schematic diagram of two-dimensional multiradar fusion imaging based on vectorization processing is shown in Figure 2. The red rectangles and blue rectangles represent the basis matrix corresponding to radar 1 and radar 2, respectively.

\section{FLBIUB Algorithm}

Large-scale data reconstruction is involved in solving equation (12). It is necessary to find an effective and efficient block sparse reconstruction algorithm to achieve multiradar ISAR fusion imaging of block structure targets. Considering the block sparsity in the echo data of block structure targets, the sparse representation problem in equation (12) can be transformed into an unconstrained block sparsity optimization problem. Based on the LBI algorithm [16], the FLBIUB algorithm is proposed to solve the block sparse signal reconstruction problem in equation (12). The weighted back-adding residual and the condition number optimization of the basis matrix are combined to further accelerate the iteration convergence speed.

3.1. Solution Process with Back-Adding Residual. As for the block structure targets, the target image vector $\tilde{a}$ can be

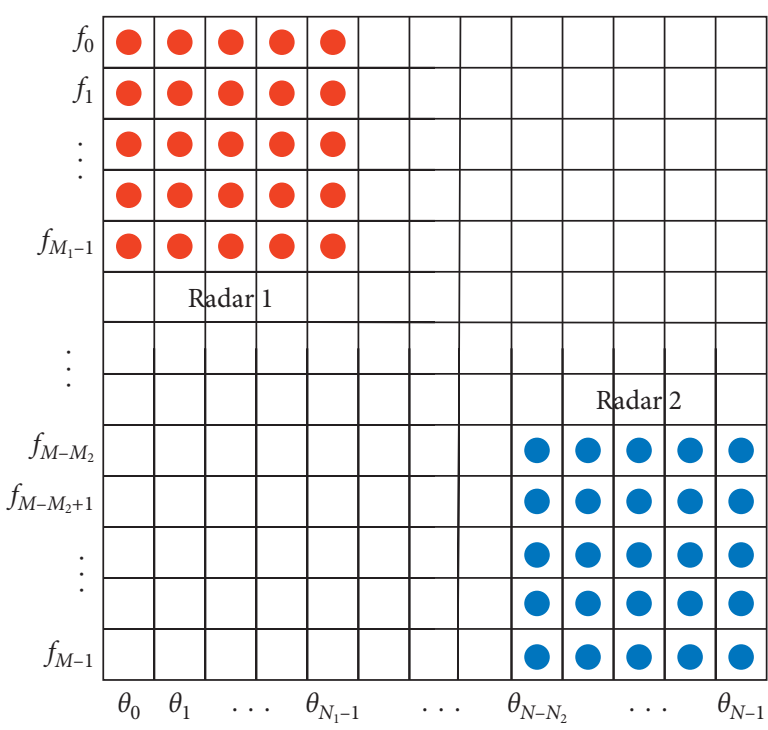

FIGURE 1: The observation model of the two radars for ISAR fusion imaging.

regarded as a block sparse signal. Assuming that $\tilde{a}$ can be divided into $B$ blocks, which can be expressed as

$$
\widetilde{a}=[\underbrace{\widetilde{a}_{1}, \ldots, \widetilde{a}_{d_{1}}}_{d_{1}}, \underbrace{\tilde{a}_{d_{1}+1}, \ldots, \widetilde{a}_{d_{1}+d_{2}}}_{d_{2}}, \ldots, \underbrace{\tilde{a}_{5 K L-d_{B}+1}, \ldots, \widetilde{a}_{5 K L}}_{d_{B}}]^{T},
$$

where $5 \mathrm{KL}=\sum_{i=1}^{B} d_{i}, \tilde{a}[i]$ is denoted as the $i-$ th block with the length of $d_{i}$. Considering the noise, the block sparse signal reconstruction problem in equation (12) can be transformed into the optimization problem as follows [11].

$$
\begin{aligned}
& \min \|\widetilde{a}\|_{2,0}, \\
& \text { s.t. }\|\overline{\bar{s}}-\widetilde{F} \widetilde{a}\|_{2} \leq \zeta,
\end{aligned}
$$

where $\|\widetilde{a}\|_{2,0}$ is denoted as the number of nonzero data blocks in $\tilde{a}, \zeta$ represents the noise level, and $\zeta>0$.

The optimization problem in equation (16) is an NPhard problem which is hard to be solved directly. When $\widetilde{F}$ satisfies the block-restricted isometric property [11], the solution of equation (16) can be approximated by solving the following convex optimization problem:

$$
\begin{aligned}
& \min \|\widetilde{a}\|_{2,1}, \\
& \text { s.t. }\|\bar{s}-\widetilde{F} \widetilde{a}\|_{2} \leq \zeta,
\end{aligned}
$$

or equation (16) can be equivalent to an unconstrained optimization problem as

$$
\min \left\{\|\widetilde{a}\|_{2,1}+\frac{v}{2}\|\bar{s}-\widetilde{F} \widetilde{a}\|_{2}^{2}\right\},
$$

where $\|\widetilde{a}\|_{2,1}=\sum_{i=1}^{B}\|\widetilde{a}[i]\|_{2}$, and $v>0$.

An auxiliary variable $\mathbf{z}=\widetilde{a}$ is introduced to solve the unconstrained optimization problem in equation (18), which can be equivalent to 


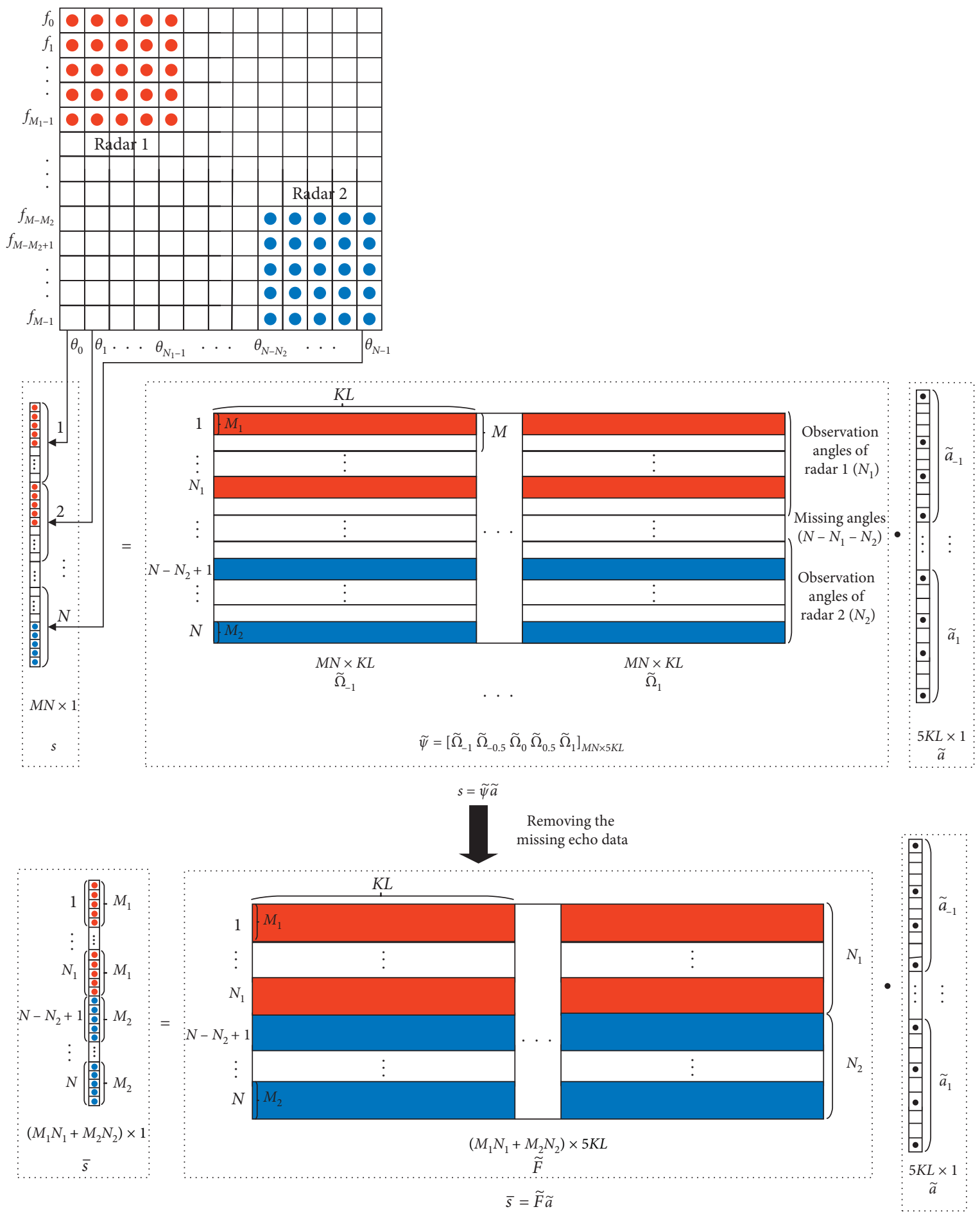

FIgURE 2: Two-dimensional multiradar fusion imaging based on vectorization processing.

$$
\begin{aligned}
& \min \left\{\|\mathbf{z}\|_{2,1}+\frac{v}{2}\|\bar{s}-\widetilde{F} \widetilde{a}\|_{2}^{2}\right\}, \\
& \text { s.t. } \mathbf{z}=\widetilde{a} .
\end{aligned}
$$

The constrained optimization problem in equation (19) can be further transformed into the following unconstrained optimization problem as

$$
\min _{\widetilde{a}, \mathbf{z}}\left\{\|\mathbf{z}\|_{2,1}+\frac{v}{2}\|\bar{s}-\widetilde{F} \widetilde{a}\|_{2}^{2}+\frac{\lambda}{2}\|\mathbf{z}-\widetilde{a}\|_{2}^{2}\right\},
$$

where $\lambda>0$.

Bregman iteration algorithm can be utilized to solve equation (20). The iteration steps are as follows: 


$$
\begin{aligned}
\left(\widetilde{a}^{g+1}, \mathbf{z}^{g+1}\right) & =\underset{\widetilde{a}, \mathbf{z}}{\arg \min }\left\{\|\mathbf{z}\|_{2,1}+\frac{v}{2}\|\bar{s}-\widetilde{F} \widetilde{a}\|_{2}^{2}+\frac{\lambda}{2}\left\|\mathbf{z}-\tilde{a}-\mathbf{b}^{g}\right\|_{2}^{2}\right\} \\
\mathbf{b}^{g+1} & =\mathbf{b}^{g}+\left(\widetilde{a}^{g+1}-\mathbf{z}^{g+1}\right) \\
\bar{s}^{g+1} & =\bar{s}^{g}+\alpha\left(\bar{s}-\widetilde{F} \widetilde{a}^{g+1}\right)
\end{aligned}
$$

where $\alpha(0 \leq \alpha<1)$ is the weighted parameter that controls the back-adding residual. By controlling the residual, the convergence speed of the iteration can be accelerated further.

Equation (21) can be transformed into the following two suboptimization problems to solve $\widetilde{a}$ and $\mathbf{z}$, respectively.

$$
\begin{aligned}
& \tilde{a}^{g+1}=\underset{\widetilde{a}}{\arg \min }\left\{\frac{v_{\|}}{2}\left\|\bar{s}^{g}-\widetilde{F} \tilde{a}\right\|_{2}^{2}+\frac{\lambda}{2}\left\|\tilde{a}-\mathbf{z}^{g}+\mathbf{b}^{g}\right\|_{2}^{2}\right\}, \\
& \mathbf{z}^{g+1}=\underset{\mathbf{z}}{\arg \min }\left\{\|\mathbf{z}\|_{2,1}+\frac{\lambda}{2}\left\|\mathbf{z}-\tilde{a}^{g+1}-\mathbf{b}^{g}\right\|_{2}^{2}\right\} .
\end{aligned}
$$

The optimization problem in equation (24) can be solved by taking the derivative of the objective function with respect to and setting it to zero; we have

$$
\left(v \widetilde{\mathbf{F}}^{H} \widetilde{\mathbf{F}}+\lambda \mathbf{I}\right) \widetilde{\mathbf{a}}-v \widetilde{\mathbf{F}}^{H} \overline{\mathbf{s}}^{\mathcal{g}}-\boldsymbol{\lambda}\left(\mathbf{z}^{g}-\mathbf{b}^{g}\right)=0 .
$$

The iterative update formula of $\widetilde{\mathbf{a}}$ can be derived from equation (26) as

$$
\widetilde{\mathbf{a}}^{g+1}=\left(v \widetilde{\mathbf{F}}^{H} \widetilde{\mathbf{F}}+\lambda \mathbf{I}\right)^{-1}\left(v \widetilde{\mathbf{F}}^{H} \overline{\mathbf{s}}^{g}+\lambda\left(\mathbf{z}^{g}-\mathbf{b}^{g}\right)\right) .
$$

The optimization problem in equation (25) can be solved by the block shrinkage operator [23], which is equivalent to

$$
z^{\mathrm{g}+1}=\underset{z}{\arg \min }\left\{\sum_{\mathrm{i}=1}^{B}\left(\|\mathbf{z}[i]\|_{2}+\frac{\lambda}{2}\left\|\mathbf{z}[i]-\widetilde{\mathbf{a}}^{\mathrm{g}+1}[i]-\mathbf{b}^{g}[i]\right\|_{2}^{2}\right)\right\} .
$$

$B$ suboptimization problems are involved in equation (28). The closed optimal solution corresponding to each block $\mathbf{z}$ [i] can be derived by the threshold shrinkage as

$$
\mathbf{z}^{\mathrm{g}+1}[i]=\operatorname{shrink}\left(\widetilde{\mathbf{a}}^{\mathrm{g}+1}[i]+\mathbf{b}^{g}[i], \frac{1}{\lambda}\right) \quad \mathrm{i}=1, \ldots, \mathrm{B},
$$

where $\operatorname{shrink}(\mathbf{x}, \gamma)=\left(\mathbf{x} /\|\mathbf{x}\|_{2}\right) \max \left\{\|\mathbf{x}\|_{2}-\gamma, 0\right\}$.

3.2. Condition Number Optimization. The convergence speed of Bregman iterative algorithms can be further improved by optimizing the condition number of the basis matrix [24]. The condition number of the basis matrix $\widetilde{\mathbf{F}}$ is defined as

$$
\operatorname{cond}(\widetilde{\mathbf{F}})=\sqrt{\frac{\lambda_{\max }\left(\widetilde{\mathbf{F}} \widetilde{\mathbf{F}}^{H}\right)}{\lambda_{\min }\left(\widetilde{\mathbf{F}} \widetilde{\mathbf{F}}^{H}\right)}}
$$

where $\lambda_{\max }\left(\widetilde{\mathbf{F}} \widetilde{\mathbf{F}}^{H}\right)$ and $\lambda_{\min }\left(\widetilde{\mathbf{F}} \widetilde{\mathbf{F}}^{H}\right)$ are the maximum and minimum eigenvalues of $\widetilde{\mathbf{F}} \widetilde{\mathbf{F}}^{H}$, respectively. The smaller the condition number, the faster the convergence speed. It can be seen from equation (30) that $\operatorname{cond}(\widetilde{\mathbf{F}}) \geq 1$. Since the basis matrix $\widetilde{\mathbf{F}}$ is a full rank matrix, the condition number can be optimized by premultiplying $\left(\widetilde{\mathbf{F}} \widetilde{\mathbf{F}}^{H}\right)^{-1 / 2}$ on both sides in equation (12) as

$$
\left(\widetilde{\mathbf{F}} \widetilde{\mathbf{F}}^{H}\right)^{-1 / 2} \overline{\boldsymbol{s}}=\left(\widetilde{\mathbf{F}} \widetilde{\mathbf{F}}^{H}\right)^{-1 / 2} \widetilde{\mathbf{F}} \widetilde{\mathbf{a}} .
$$

Define $\overline{\boldsymbol{s}}_{1}=\left(\widetilde{F} \widetilde{F}^{H}\right)^{-1 / 2} \overline{\boldsymbol{s}}$ and $\widetilde{\mathbf{F}}_{1}=\left(\widetilde{\mathbf{F}} \widetilde{\mathbf{F}}^{H}\right)^{-1 / 2} \widetilde{\mathbf{F}}$; equation (31) can be rewritten as

$$
\widetilde{\mathbf{s}}_{1}=\widetilde{\mathbf{F}}_{1} \widetilde{\mathbf{a}}
$$

Since $\quad \widetilde{\mathbf{F}}_{1} \widetilde{\mathbf{F}}_{1}^{H}=\left[\left(\widetilde{\mathbf{F}} \widetilde{\mathbf{F}}^{H}\right)^{-1 / 2} \widetilde{\mathbf{F}}\right]\left[\left(\widetilde{\mathbf{F}} \widetilde{\mathbf{F}}^{H}\right)^{-1 / 2} \widetilde{\mathbf{F}}\right]^{H}=\mathbf{I}$, the condition number of $\widetilde{\mathbf{F}}_{1}$ is

$$
\operatorname{cond}\left(\widetilde{\mathbf{F}}_{1}\right)=\sqrt{\frac{\lambda_{\max }\left(\widetilde{\mathbf{F}}_{1} \widetilde{\mathbf{F}}_{1}^{H}\right)}{\lambda_{\min }\left(\widetilde{\mathbf{F}}_{1} \widetilde{\mathbf{F}}_{1}^{H}\right)}}=1 .
$$

The condition number in equation (33) is the minimum. The iterative convergence speed can be accelerated by the condition number optimization. Similar to the derivation process in Section 3.1, the iterative update formulas of the FLBIUB algorithm can be derived as

$$
\left\{\begin{array}{l}
\widetilde{\mathbf{a}}^{g+1}=\left(v \widetilde{\mathbf{F}}^{+} \widetilde{\mathbf{F}}+\lambda \mathbf{I}\right)^{-1}\left(v \widetilde{\mathbf{F}}^{+} \overline{\mathbf{s}}^{g}+\lambda\left(\mathbf{z}^{g}-\widetilde{\mathbf{F}} \mathbf{b}^{g}\right)\right), \\
\mathbf{z}^{g+1}[i]=\operatorname{shrink}\left(\widetilde{\mathbf{a}}^{g+1}[i]+\mathbf{b}^{g}[i], \frac{1}{\lambda}\right) \quad i=1, \ldots, B, \\
\mathbf{b}^{g+1}=\mathbf{b}^{g}+\left(\widetilde{\mathbf{a}}^{g+1}-\mathbf{z}^{g+1}\right), \\
\overline{\mathbf{s}}^{g+1}=\overline{\mathbf{s}}^{g}+\alpha\left(\overline{\mathbf{s}}-\widetilde{\mathbf{F}} \widetilde{\mathbf{a}}^{g+1}\right),
\end{array}\right.
$$

where $\widetilde{\mathbf{F}}^{+}=\widetilde{\mathbf{F}}^{H}\left(\widetilde{\mathbf{F}} \widetilde{\mathbf{F}}^{H}\right)^{-1}$.

Since $\left(v \widetilde{\mathbf{F}}^{+} \widetilde{\mathbf{F}}+\lambda \mathbf{I}\right)^{-1}$ needs to be calculated in each iteration when updating $\widetilde{\mathbf{a}}$, the computational complexity is heavy due to deriving the inversion of the large-scale matrix. Considering that $\widetilde{\mathbf{F}}$ is given, the matrix inversion $\left(v \widetilde{\mathbf{F}}^{+} \widetilde{\mathbf{F}}+\lambda \mathbf{I}\right)^{-1}$ can be calculated only once outside the iterative process. To further reduce the computational complexity of matrix inversion, the Woodbury formula can be utilized to transform it into

$$
\left(v \widetilde{\mathbf{F}}^{+} \widetilde{\mathbf{F}}+\lambda \mathbf{I}\right)^{-1}=\frac{1}{\lambda} \mathbf{I}-\frac{v}{\lambda} \widetilde{\mathbf{F}}^{+}\left(v \widetilde{\mathbf{F}} \widetilde{\mathbf{F}}^{+}+\lambda \mathbf{I}\right)^{-1} \widetilde{\mathbf{F}}
$$

It can be seen from equation (35) that a matrix inversion problem with the size of $5 \mathrm{KL} \times 5 \mathrm{KL}$ can be transformed into a matrix inversion problem with the size of $\left(M_{1} N_{1}+M_{2} N_{2}\right) \times\left(M_{1} N_{1}+M_{2} N_{2}\right)$ after using the Woodbury formula. Since the matrix dimension is greatly reduced, the computational complexity is also reduced remarkably. 
3.3. Implementation Process. Specifical steps of the proposed FLBIUB algorithm can be summarized in Algorithm 1.

A flowchart of the two-dimensional multiradar ISAR fusion imaging of block structure targets based on the FLBIUB algorithm is shown in Figure 3. Specifically, the steps are as follows:

Step 1: perform preprocessing such as motion compensation and mutual coherent compensation to obtain coherent echo data of each radar in range frequencyazimuth slow time domain

Step 2: discretize the echo data and establish the image model based on sparse representation

Step 3: vectorize and splice the echo data of each radar to obtain the observation data $\overline{\mathbf{s}}$ and the corresponding basis matrix $\widetilde{\mathbf{F}}$

Step 4: utilize the FLBIUB algorithm to reconstruct the signal and obtain the vector of image estimation $\widehat{\widetilde{\mathbf{a}}}$

Step 5: convert the vector $\widehat{\widetilde{\mathbf{a}}}$ into a two-dimensional matrix $\widehat{\mathbf{A}}$, which is also the target image obtained by the multiradar fusion imaging

\section{Simulation Results and Discussion}

In this section, the reconstruction performance of the FLBIUB algorithm is verified by the one-dimensional block sparse signal. The two-dimensional multiradar fusion imaging performance based on the FLBIUB algorithm is further verified by the real measurement data. The simulation experiments are all implemented in MATLAB R2017b software on a personal computer with an Intel ${ }^{\circledR}$ Core $^{\mathrm{TM}}$ i5$8265 \mathrm{U} 1.60 \mathrm{GHz}$ central processing unit (CPU) and $16 \mathrm{~GB}$ memory.

\subsection{Experiments of One-Dimensional Block Sparse Signal} Reconstruction. Assuming that the one-dimensional generated original signal $\tilde{\mathbf{a}}$ is a block sparse signal with the size of $N \times 1 . K$ nonzero elements with random amplitudes are in the signal. The nonzero elements can be divided into $B$ blocks with random lengths, and the position of each block is randomly distributed in the signal. $\widetilde{\mathbf{F}}$ is a random measurement matrix with the size of $M \times N$. The coefficients in $\widetilde{\mathbf{F}}$ obey the standard normal distribution and the column normalization. The parameters are set as $N=100, M=60$, $K=24$, and $B=4$. The generated original signal and the observation signal are shown in Figures 4(a) and 4(b), respectively.

4.1.1. The Verification of the Effectiveness. Orthogonal matching pursuit (OMP) algorithm [25], FLBI algorithm [17], BOMP algorithm [11], and FLBIUB algorithm are utilized to reconstruct the block sparse signal, respectively. The parameters are set as $v=5\left\|\widetilde{\mathbf{F}}^{+} \overline{\mathbf{s}}\right\|_{\infty}, \lambda=0.7$, and $\alpha=0.9$ in the FLBIUB algorithm. The termination criterion of the FLBIUB algorithm is $\|\widetilde{\mathbf{F}} \widehat{\tilde{a}}-\overline{\mathbf{s}}\|_{2} /\|\overline{\mathbf{s}}\|_{2} \leq 10^{-5}$ or the iteration number reaches 500 . The reconstruction time and the relative reconstruction error are utilized to evaluate the reconstruction performance, where the relative reconstruction error is defined as Err $=\|\widehat{\widetilde{\mathbf{a}}}-\widetilde{\mathbf{a}}\|_{2} /\|\widetilde{\mathbf{a}}\|_{2}$. The reconstruction results of the four algorithms are shown in Figure 5 .

It can be seen from the results that the OMP algorithm and FLBI algorithm cannot reconstruct the block sparse signal accurately. The reason is that the correlation of the block sparse signal is not considered in the OMP algorithm and FLBI algorithm. The two algorithms are not suitable for the reconstruction of block sparse signals. While the FLBIUB algorithm and BOMP algorithm can both achieve the accurate reconstruction of the block sparse signal. The relative reconstruction error of the FLBIUB algorithm is smaller than that of the BOMP algorithm. It indicates that the reconstruction accuracy of the FLBIUB algorithm is higher than the BOMP algorithm. The reconstruction times of the FLBIUB algorithm and BOMP algorithm are both small, indicating that the two algorithms can both achieve signal reconstruction quickly.

4.1.2. The Influence of the Parameters. This section mainly analyzes the influence of the parameters $v$ and $\alpha$ on the reconstruction performance of the FLBIUB algorithm.

To analyze the influence of $v$ on the reconstruction performance, we set other parameters such as $\alpha=0.5$ and $\lambda=0.7$. Let $x=\left\|\widetilde{\mathbf{F}}^{+} \overline{\mathbf{s}}\right\|_{\infty}$; FLBIUB algorithm with different $v$ is utilized to reconstruct the block sparse signal. The termination criterion is $\|\widetilde{\mathbf{F}} \widehat{\tilde{a}}-\overline{\mathbf{s}}\|_{2} /\|\overline{\mathbf{s}}\|_{2} \leq 10^{-5}$ or the iteration number reaches 200 . The relative reconstruction error versus iterations number with different $v$ is shown in Figure 6. It can be seen that the algorithm converges slowly and requires more iterations when $v$ is smaller (for example, $v=0.1 x$ ). The convergence speed improves when $v$ increases, and the number of iterations required to reach the convergence is reduced. However, the reconstruction error is also increased. Hence, the reconstruction accuracy and the convergence speed should be both considered to set the appropriate value of $v$, which cannot be set too small or too large. Generally, it is a more appropriate set as $v=5\left\|\widetilde{\mathbf{F}}^{+} \overline{\mathbf{s}}\right\|_{\infty}$.

To analyze the influence of $\lambda$ on the reconstruction performance, we set other parameters such as $\alpha=0.5$ and $v=5\left\|\widetilde{\mathbf{F}}^{+} \overline{\mathbf{s}}\right\|_{\infty}$. FLBIUB algorithm with different $\lambda$ is utilized to reconstruct the block sparse signal. The termination criterion is $\|\widetilde{\mathbf{F}} \widehat{\tilde{a}}-\overline{\mathbf{s}}\|_{2} /\|\overline{\mathbf{s}}\|_{2} \leq 10^{-5}$ or the iteration number reaches 200 . The relative reconstruction error versus iterations number with different $\lambda$ is shown in Figure 7 . It can be seen that the algorithm requires fewer iterations to reach convergence and the reconstruction error is higher when $\lambda$ is smaller (for example, $\lambda=0.1$ ). The convergence speed decreases and the number of iterations required to reach convergence increases when $\lambda$ increases. However, the reconstruction error is decreased and the reconstruction accuracy is improved as $\lambda$ increases. Hence, the reconstruction accuracy and the convergence speed should be both considered to set the appropriate value of $\lambda$ according to specific requirements.

To analyze the influence of $\alpha$ on the reconstruction performance, we set other parameters such as $v=5\left\|\widetilde{\mathbf{F}}^{+} \overline{\mathbf{s}}\right\|_{\infty}$ 
FLBIUB algorithm.

Input: $\overline{\mathbf{s}}, \widetilde{\mathbf{F}}$.

Initialize: $\widetilde{\mathbf{a}}^{0}=\mathbf{Z}^{0}=\mathbf{b}^{0}=0, v>0, \lambda>0, g=0, \widetilde{\mathbf{F}}^{+}=\widetilde{\mathbf{F}}^{H}\left(\widetilde{\mathbf{F}} \widetilde{\mathbf{F}}^{H}\right)^{-1}$.

Iteration process: Determine whether to terminate the iteration. If the iteration termination condition is satisfied, output the result $\widehat{\tilde{a}}$; otherwise, continue to perform the following iteration process as

$\widetilde{\mathbf{a}}^{g+1}=\left(v \widetilde{\mathbf{F}}^{+} \widetilde{\mathbf{F}}+\lambda \mathbf{I}\right)^{-1}\left(v \widetilde{\mathbf{F}}^{+} \overline{\mathbf{s}}^{g}+\lambda\left(\mathbf{z}^{g}-\mathbf{b}^{g}\right)\right)$

$\mathbf{z}^{g+1}[i]=\operatorname{shrink}\left(\widetilde{\mathbf{a}}^{g+1}[i]+\mathbf{b}^{g}[i], 1 / \lambda\right) \quad i=1, \ldots, B$

$\mathbf{b}^{g+1}=\mathbf{b}^{g}+\left(\widetilde{\mathbf{a}}^{g+1}-\mathbf{z}^{g+1}\right)$

$\overline{\mathbf{s}}^{g+1}=\overline{\mathbf{s}}^{g}+\alpha\left(\bar{s}-\widetilde{\mathbf{F}} \widetilde{\mathbf{a}}^{g+1}\right)$

$g=g+1$

Output: $\widehat{\widetilde{\mathbf{a}}}=\widetilde{\mathbf{a}}^{g}$.

Algorithm 1: Specifically steps of the FLBIUB algorithm.

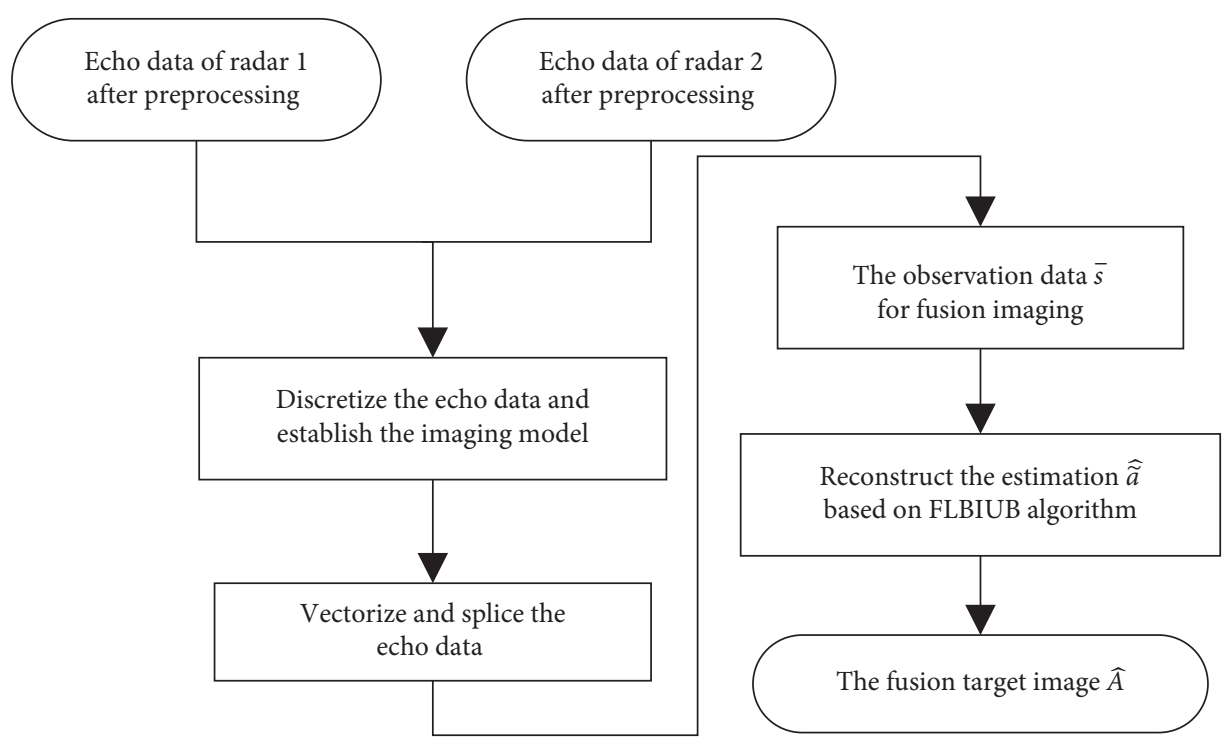

FIGURE 3: Flowchart of the two-dimensional multiradar ISAR fusion imaging.

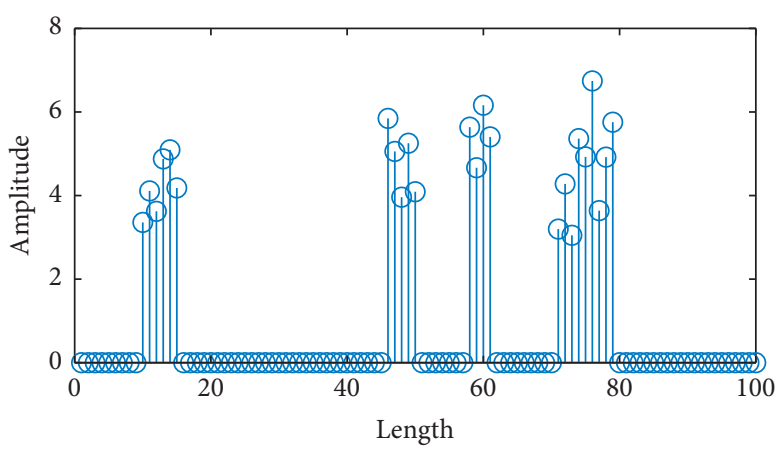

(a)

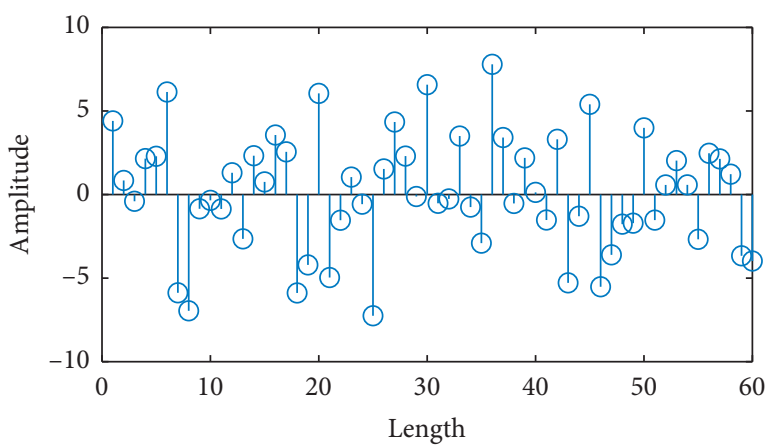

$\odot$ Observation signal

(b)

Figure 4: The original signal and the observation signal. (a) Original signal. (b) Observation signal. 


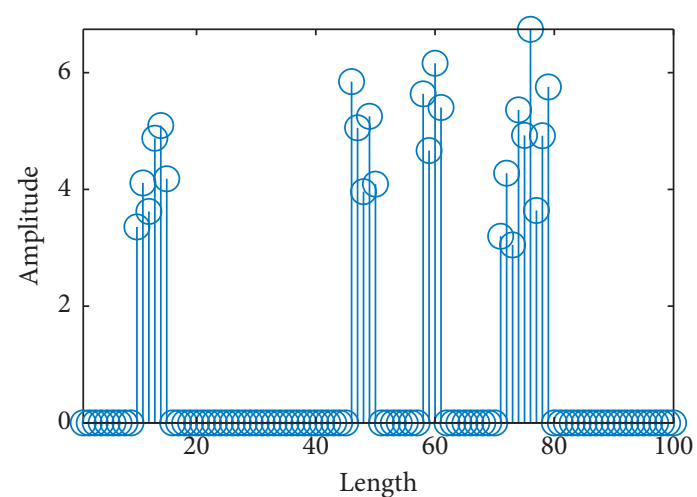

Err $=6.03 e-06$, Time $=0.00155 \mathrm{~s}$

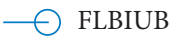

(a)

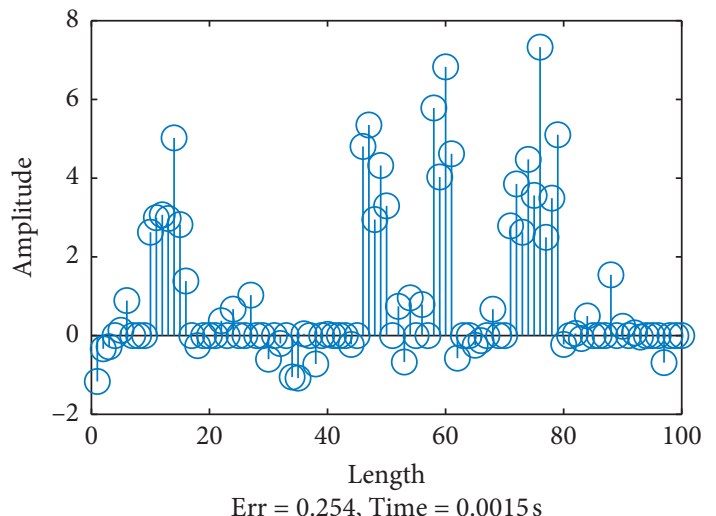

$\bigcirc$ FLBI

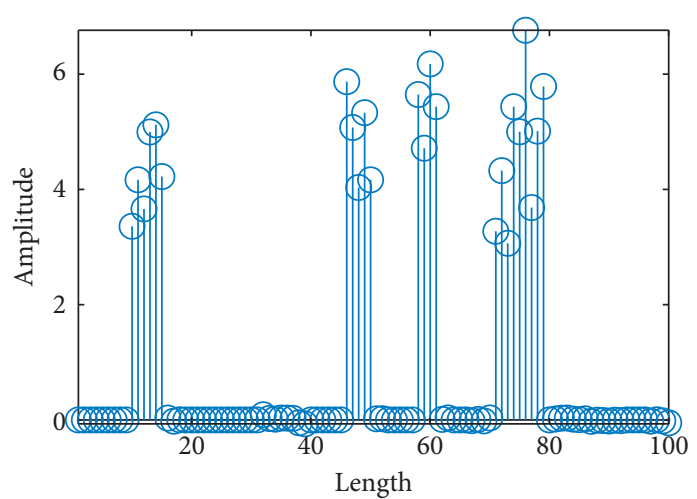

Err $=0.0138$, Time $=0.00117 \mathrm{~s}$

$\bigcirc$ BOMP

(b)

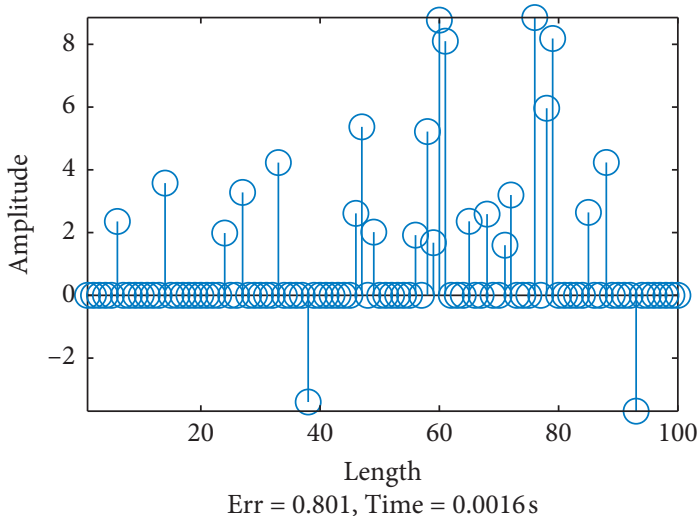

$\bigcirc \mathrm{OMP}$

(c)

(d)

FIGURE 5: Block sparse signal reconstruction results of the respective algorithms. (a) FLBIUB algorithm. (b) BOMP algorithm. (c) FLBI algorithm. (d) OMP algorithm.

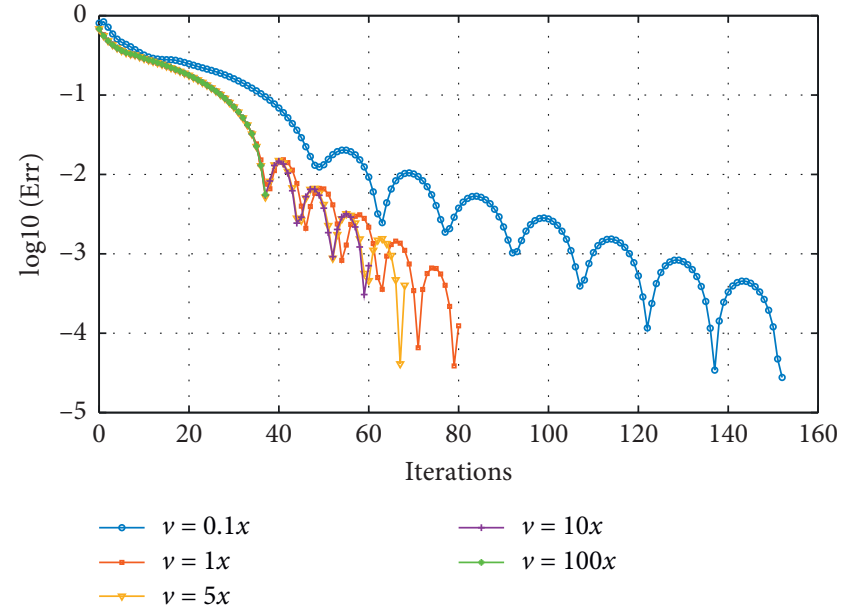

FIGURE 6: Relative reconstruction error versus iterations number with different $v$.

and $\lambda=0.7$. FLBIUB algorithm with different $\alpha$ is utilized to reconstruct the block sparse signal. The termination criterion is $\|\widetilde{\mathbf{F}} \widehat{\widehat{\mathbf{a}}}-\overline{\mathbf{s}}\|_{2} /\|\overline{\mathbf{s}}\|_{2} \leq 10^{-5}$ or the iteration number reaches
200. The relative reconstruction error versus iterations number with different $\alpha$ is shown in Figure 8. It can be seen that the algorithm requires more iterations to reach convergence and the reconstruction error is high when $\alpha=0$. The reason is that the back-adding residual is not considered in the algorithm when $\alpha=0$. The convergence speed improves and the number of iterations required to reach convergence decreases when $\alpha$ increases. It indicates that considering the back-adding residual can further improve the iterative convergence speed. However, the reconstruction error also increases. Hence, the reconstruction accuracy and the convergence speed should be both considered to set the appropriate value of $\alpha$ within $[0,1)$.

4.1.3. The Influence of the Sampling Rate and the Signal Sparsity. This section mainly analyzes the influence of the sampling rate and signal sparsity on the reconstruction performance of the proposed algorithm. The sampling rate is defined as the ratio of the observation signal length to the original signal length, which can be expressed as $\rho=M / N$. The signal sparsity $K$ represents the number of nonzero elements in the sparse signal. 


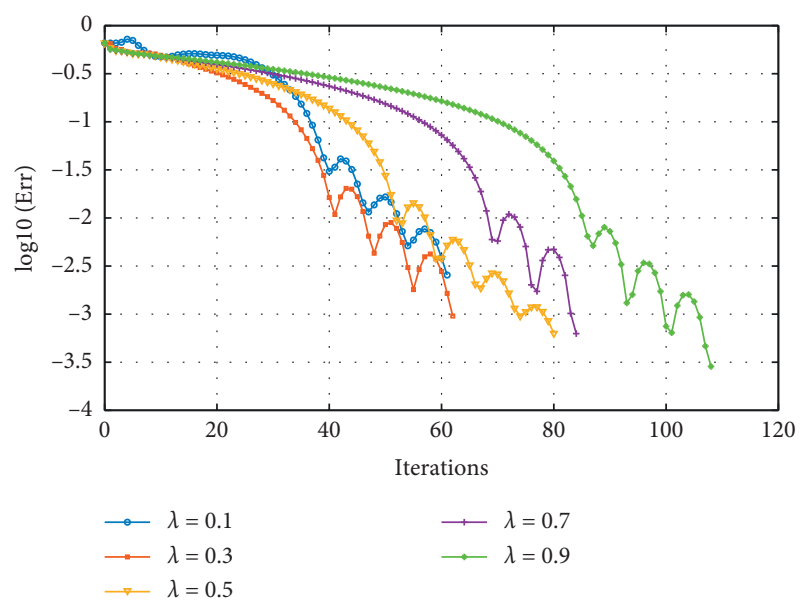

FIGURE 7: Relative reconstruction error versus iterations number with different $\lambda$.

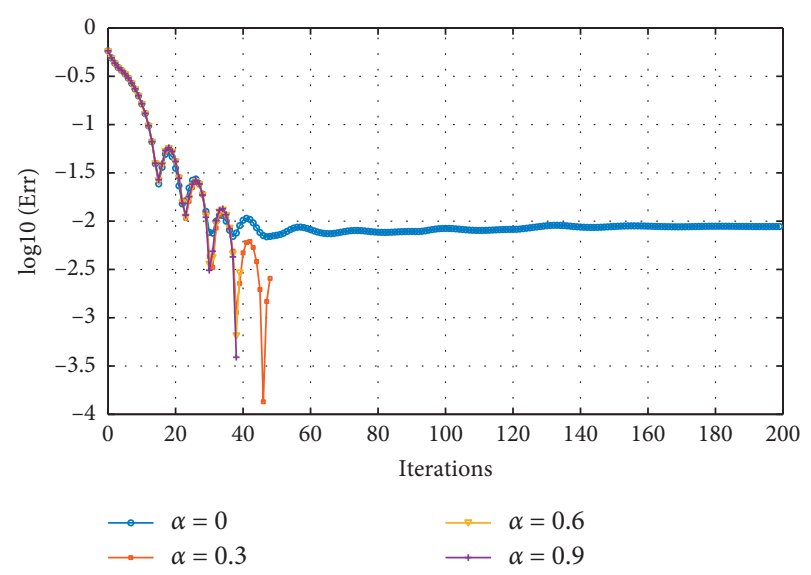

FIGURE 8: Relative reconstruction error versus iteration number with different $\alpha$.

The influence of the sampling rate on the reconstruction performance is analyzed by varying the length of the observation signal. The signal sparsity is set as $K=24$, and the length of the original signal is set as $N=100$. The length of the observation signal $M$ ranges from 40 to 80 with a step size of 5 . BOMP algorithm and FLBIUB algorithm are utilized to reconstruct the block sparse signal, respectively. The parameters in the FLBIUB algorithm are set as $v=5\left\|\widetilde{\mathbf{F}}^{+} \overline{\mathbf{s}}\right\|_{\infty}, \alpha=0.6$, and $\lambda=0.5$. The termination criterion of the FLBIUB algorithm is $\|\widetilde{\mathbf{a}} \hat{\mathbf{a}}-\overline{\mathbf{s}}\|_{2} /\|\overline{\mathbf{s}}\|_{2} \leq 10^{-5}$ or the iteration number reaches 200.100 independent Monte Carlo trials have been conducted to reconstruct the block sparse signal and obtain the estimation $\widetilde{\mathbf{a}}$ under each fixed $M$. A trail is considered as a successful one when $\|\widetilde{\tilde{\mathbf{a}}}-\widetilde{\mathbf{a}}\|_{2}^{2} /\|\widetilde{\mathbf{a}}\|_{2}^{2} \leq 10^{-3}$. The reconstruction success rate is defined as the percentage of the number of successful reconstruction trails to the total number of tails. The success rate of the respective algorithms versus sampling rate is shown in Figure 9(a). It can be seen from Figure 9(a) that the reconstruction success rate increases with the increase of the sampling rate. It indicates that the more observations, the better the reconstruction of the sparse signal. Moreover, the FLBIUB algorithm has a higher reconstruction success rate than the BOMP algorithm with the same sampling rate. It indicates that the FLBIUB algorithm can reconstruct the sparse signal better under a low sampling rate.

The influence of the signal sparsity $K$ on the reconstruction performance is analyzed by varying the number of sparsity. The length of the original signal is set as $N=100$, and the length of the observation signal is set as $M=60$. The signal sparsity $K$ ranges from 5 to 50 with the step size 5 . BOMP algorithm and FLBIUB algorithm are utilized to reconstruct the block sparse signal, respectively. The parameters in the FLBIUB algorithm are set as $v=5\left\|\widetilde{\mathbf{F}}^{+}\right\|_{\infty}$, $\alpha=0.6$, and $\lambda=0.5$. The termination criterion of the FLBIUB algorithm is $\|\widetilde{\mathbf{F}} \widetilde{\widetilde{a}}-\overline{\mathbf{s}}\|_{2} /\|\overline{\mathbf{s}}\|_{2} \leq 10^{-5}$ or the iteration number reaches 200. 100 independent Monte Carlo trials have been conducted to reconstruct the block sparse data under each fixed $K$. The success rate of the respective algorithms versus sparsity is shown in Figure 9(b). It can be seen from Figure 9(b) that the success rate decreases with the increase of sparsity $K$. It indicates that the smaller the sparsity, the better the reconstruction of the sparse signal. Moreover, the FLBIUB algorithm has a higher success rate than the BOMP algorithm with the same signal sparsity. It indicates that the FLBIUB algorithm can reconstruct the sparse signal better within a larger sparsity range and wider application than the BOMP algorithm.

4.1.4. The Verification of the Antinoise Performance. Set the parameters as $N=100, M=60$, and $K=24$ to generate the original sparse signal and the observation signal. To verify the antinoise performance of the proposed algorithm, white Gaussian noise is added to the observation signal. BOMP algorithm and FLBIUB algorithm are utilized to reconstruct the block sparse signal, respectively. The normalized mean square error (NMSE) is utilized to evaluate the reconstruction performance. NMSE is defined as NMSE $=\|\widehat{\widetilde{\mathbf{a}}}-\widetilde{\mathbf{a}}\|_{2}^{2} /\|\widetilde{\mathbf{a}}\|_{2}^{2}$. The smaller the NMSE, the higher the reconstruction accuracy. The parameters in the FLBIUB algorithm are set as $v=5\left\|\widetilde{\mathbf{F}}^{+} \overline{\mathbf{s}}\right\|_{\infty}, \alpha=0.6$, and $\lambda=0.5$. The termination criterion of the FLBIUB algorithm is $\|\widetilde{\mathbf{F}} \widehat{\vec{a}}-\overline{\mathbf{s}}\|_{2} \leq M \sigma^{2}$ or the iteration number reaches 200 , where $\sigma^{2}$ is the noise variance. The signal-to-noise ratio (SNR) ranges from $0 \mathrm{~dB}$ to $30 \mathrm{~dB}$ with a step size of $5 \mathrm{~dB} .100$ independent Monte Carlo trials have been conducted to reconstruct the block sparse data under each fixed SNR. The NMSE is averaged over 100 tails. The NMSE of the respective algorithms versus SNR is shown in Figure 10.

It can be seen that the NMSE of the FLBIUB algorithm is very small when SNR is higher than $15 \mathrm{~dB}$. It indicates that the block sparse signal can be reconstructed accurately by the FLBIUB algorithm under a high SNR condition. The NMSE of the two algorithms increases as SNR decreases. The reason is that high noise will affect the reconstruction performance of the algorithms under a low SNR condition. However, the NMSE of the FLBIUB algorithm is lower than the BOMP algorithm under the same SNR condition. It illustrates that the FLBIUB algorithm has better antinoise performance than the BOMP algorithm in block sparse signal reconstruction. 


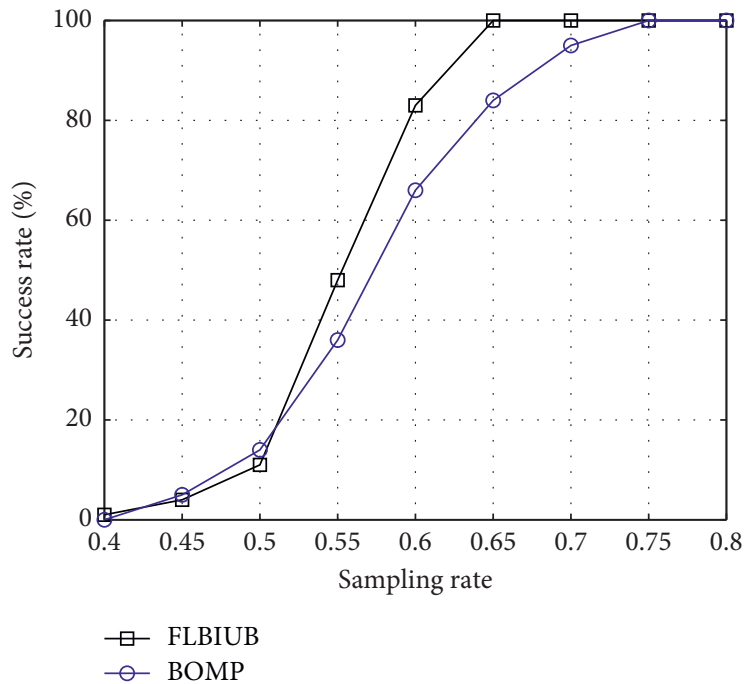

(a)

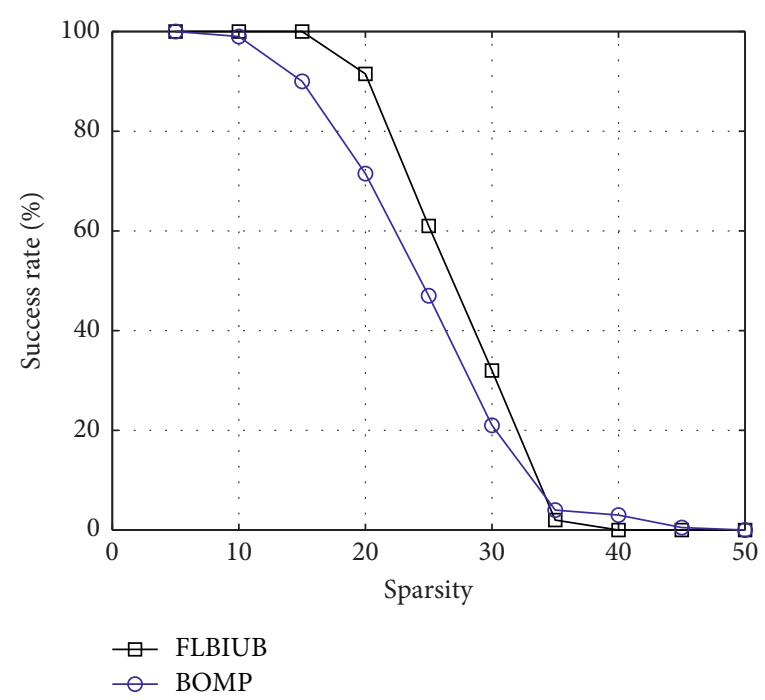

(b)

Figure 9: The success rate of the respective algorithms. (a) Success rate versus sampling rate. (b) Success rate versus sparsity.

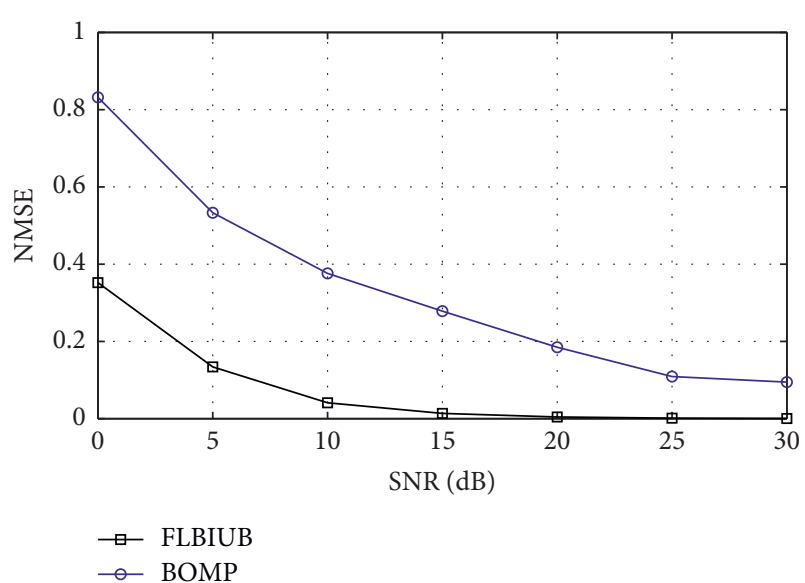

FIGURE 10: The NMSE of the respective algorithms versus SNR.

\subsection{Experiments of Two-Dimensional ISAR Fusion Imaging.} In practical scenes, aircraft targets with complex structures can be approximated as block structure targets. To verify the two-dimensional fusion imaging performance of the proposed algorithm for block structure targets, the measured data of Yak-42 aircraft is exploited to achieve the two-dimensional multiradar ISAR fusion imaging. The carrier frequency is $5.52 \mathrm{GHz}$, the bandwidth is $400 \mathrm{MHz}$, the pulse repetition frequency is $100 \mathrm{~Hz}$, the pulse width is $25.6 \mathrm{us}$, and the sampling frequency is $10 \mathrm{MHz}$. The size of the full-band and full-angle echo data is $200 \times 200$. The two-dimensional echo data and the range-Doppler (RD) imaging result of the full-band and full-angle echo data are shown in Figures 11(a) and $11(\mathrm{~b})$, respectively.

The sizes of $M_{1} \times N_{1}$ and $M_{2} \times N_{2}$ are selected from the upper left and lower right corners of the full-band and fullangle echo data as the echo data of radar 1 and radar 2, respectively. The parameters are set as $M_{1}=M_{2}=60$ and $N_{1}=N_{2}=60$. White Gaussian noise is added to the echo data, and the SNR is $20 \mathrm{~dB}$. The RD imaging results of radar 1 and radar 2 are shown in Figures 12(a) and 12(b), respectively. Due to the limited bandwidth and observation angle, the two-dimensional imaging resolution of monostatic radar is poor. The outline of the aircraft in the imaging results of the two radars is not clear. The ISAR fusion imaging result obtained by the RD algorithm is shown in Figure 12(c). Compared with the imaging result of monostatic radar, the resolution is improved after the fusion imaging based on the $\mathrm{RD}$ algorithm. Some detailed structural information can be seen in Figure 12(c). However, due to the missing frequency band and observation angle in the observation echo data, strong sidelobes and energy leakage occur in the FFT compression imaging process with $\mathrm{RD}$ algorithm, which affects the imaging quality.

The two-dimensional observation echo data of the two radars is shown in Figure 13(a). FLBI algorithm, BOMP algorithm, and FLBIUB algorithm are utilized to achieve multiradar fusion imaging. The fusion imaging results of the respective algorithms are shown in Figures 13(b)-13(d), respectively. It can be seen from Figure 13(b) that the basic outline of the aircraft can be obtained by the FLBI algorithm. However, some scatterers are missing in the result, and the block structure characteristics of the target are not obvious in the fusion image. Since the block sparsity of the signal is not considered in the FLBI algorithm, the block clustering effect is not obvious in the reconstruction result. It can be seen from Figure 13(c) that the block clustering effect is in the fusion imaging results obtained by the BOMP algorithm. However, some scatterers are missing, and some false scatters are introduced in the fusion imaging result, which affects the recognition of the overall shape of the aircraft. It can be seen from Figure 13(d) that the outline of the aircraft in the fusion imaging result obtained by the FLBIUB algorithm is clean, the block structure characteristics of the target are obvious, and the overall shape of the target is clear to be distinguished. 


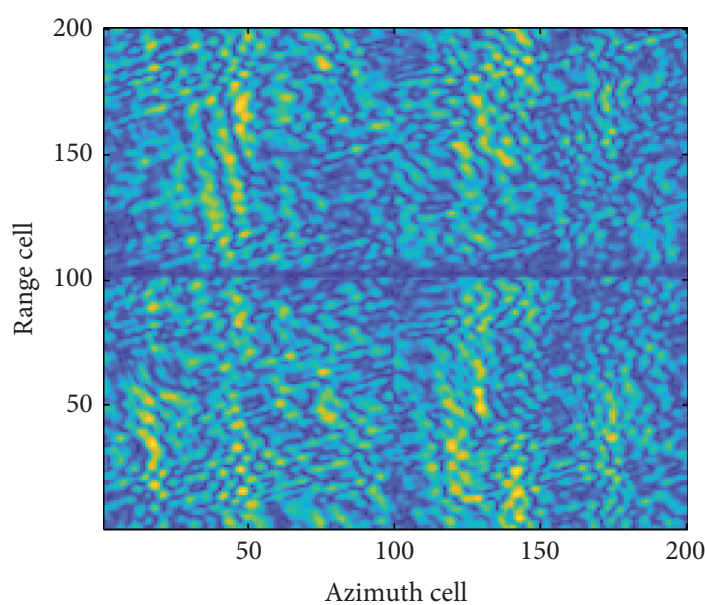

(a)

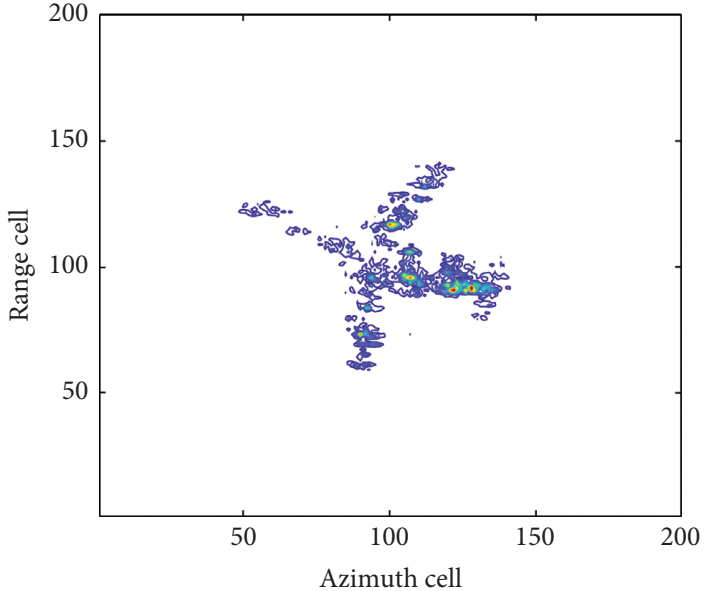

(b)

FIgURE 11: Two-dimensional echo data and RD imaging result of the full-band and full-angle echo data. (a) Two-dimensional echo data. (b) RD imaging result.

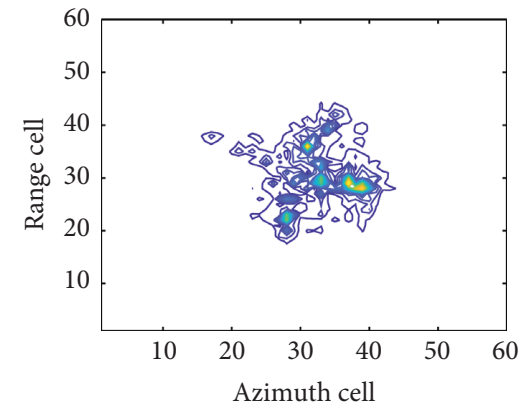

(a)

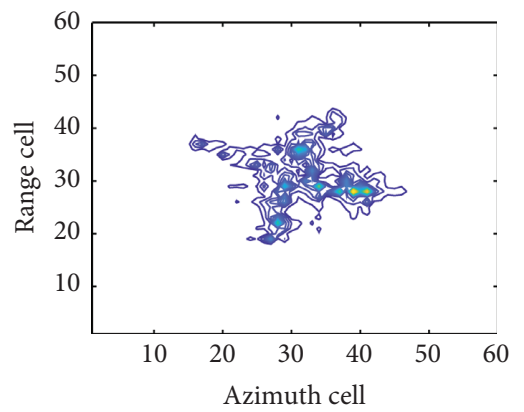

(b)

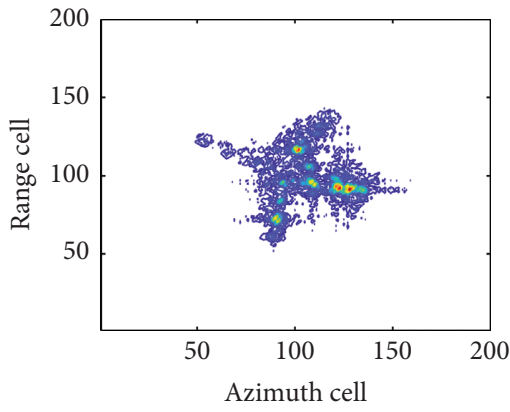

(c)

FiguRe 12: RD imaging results. (a) RD image of radar 1. (b) RD image of radar 2. (c) RD fusion image of two radars.

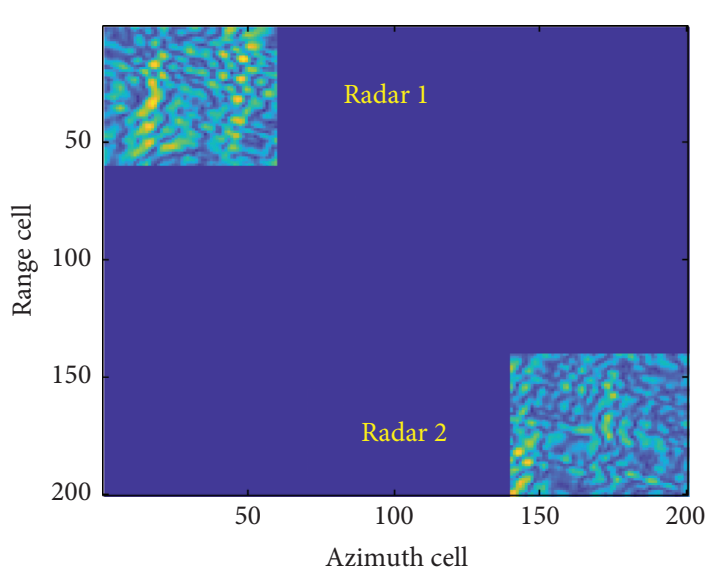

(a)

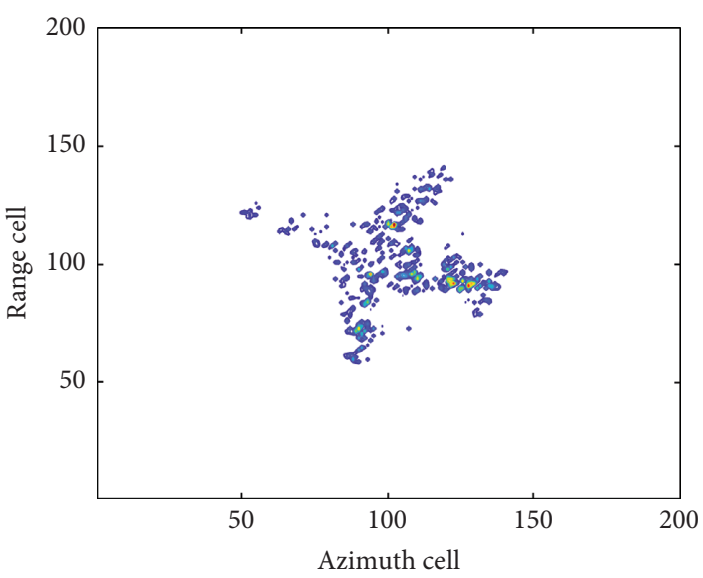

(b)

FIGURE 13: Continued. 


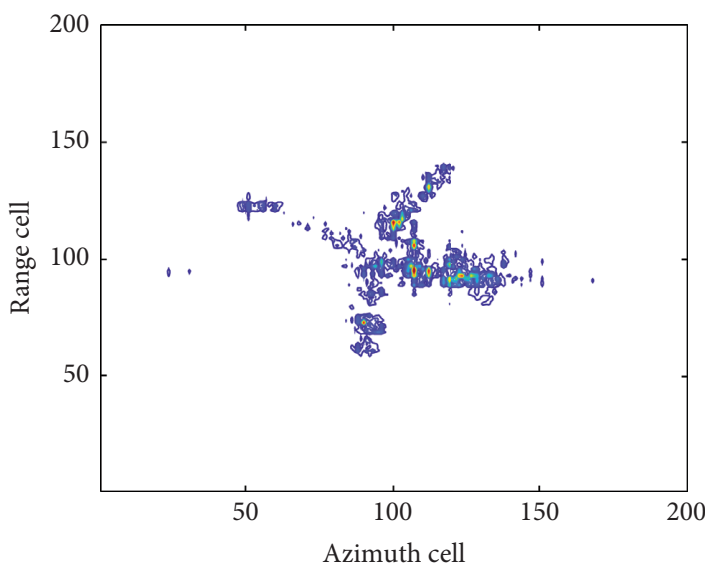

(c)

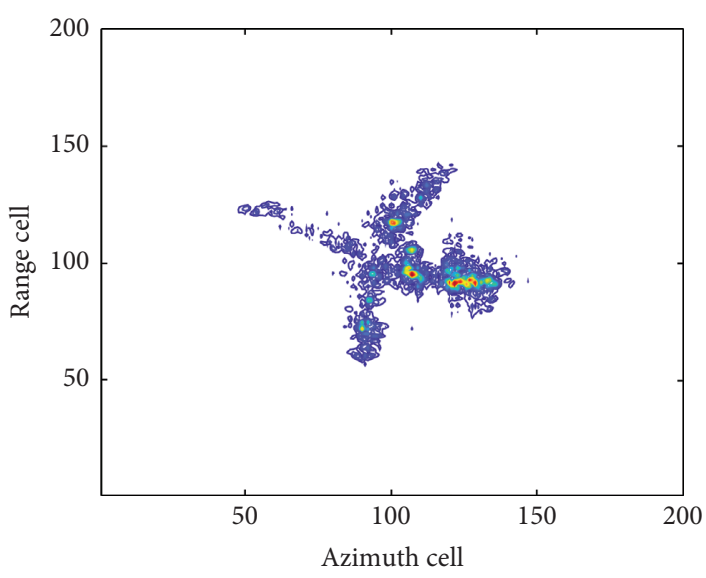

(d)

Figure 13: Two-dimensional observation echo data and fusion imaging results with $M_{1}=M_{2}=60$ and $N_{1}=N_{2}=60$. (a) Two-dimensional observation echo data. (b) Fusion imaging results of FLBI algorithm. (c) Fusion imaging results of BOMP algorithm. (d) Fusion imaging results of FLBIUB algorithm.

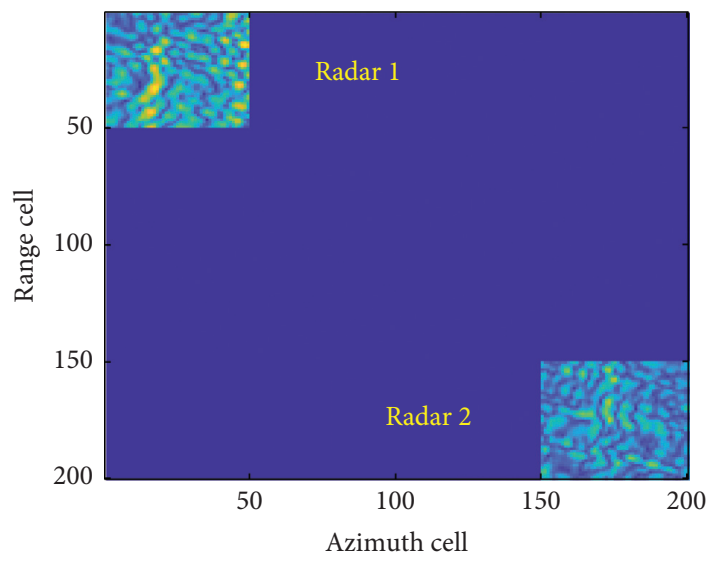

(a)

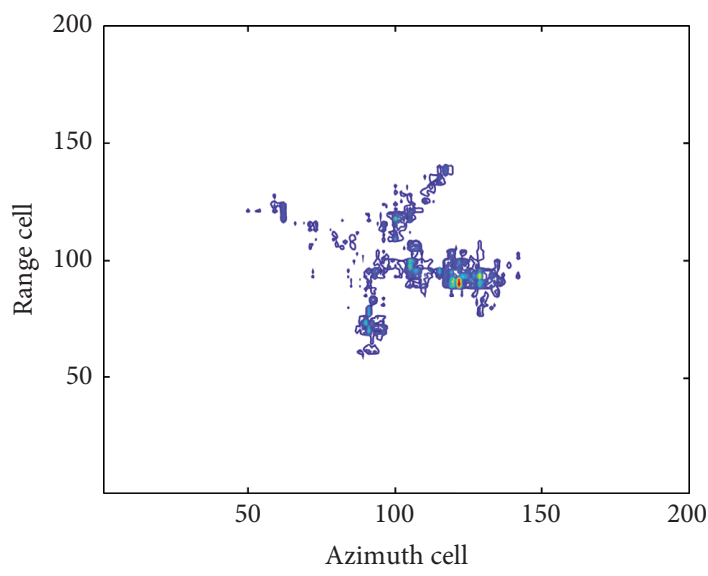

(c)

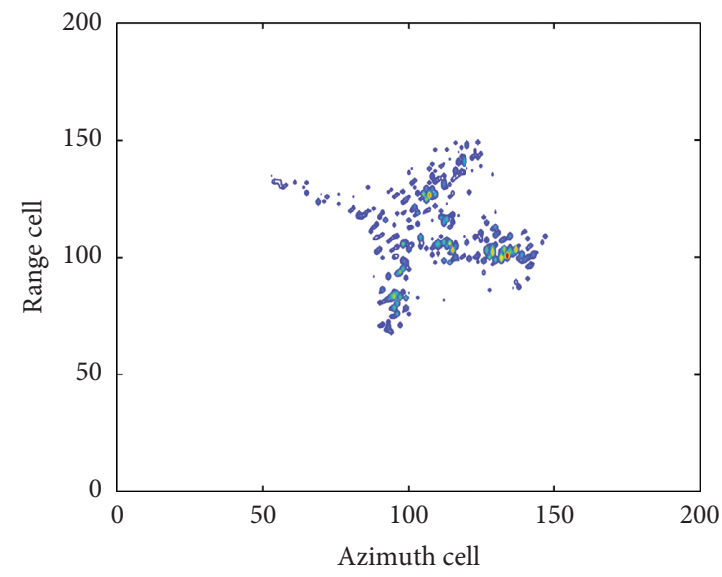

(b)

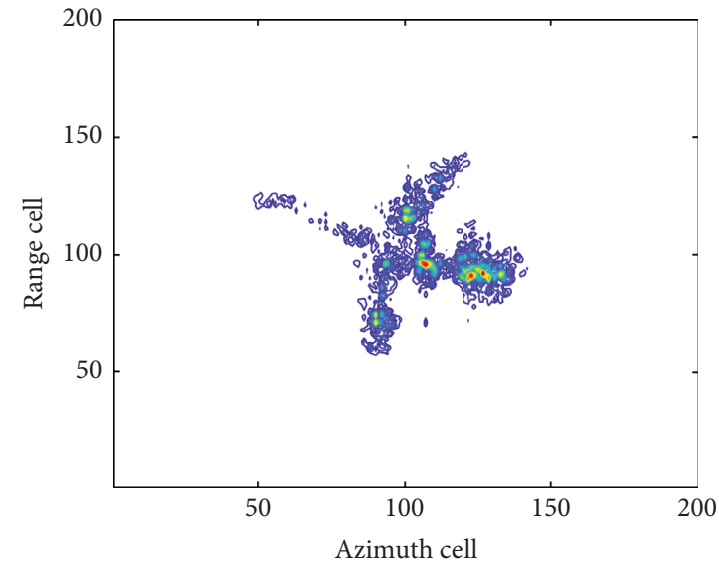

(d)

Figure 14: Two-dimensional observation echo data and fusion imaging results with $M_{1}=M_{2}=50$ and $N_{1}=N_{2}=50$. (a) Two-dimensional observation echo data. (b) Fusion imaging results of FLBI algorithm. (c) Fusion imaging results of BOMP algorithm. (d) Fusion imaging results of FLBIUB algorithm. 
4.2.1. Different Observation Cases. The SNR of the observation echo data is kept at $20 \mathrm{~dB}$. The bandwidth and angle of the observation data are changed to further verify the fusion imaging performance under different observation cases.

Case 1. Let $M_{1}=M_{2}=50$ and $N_{1}=N_{2}=50$; the two-dimensional observation echo data is shown in Figure 14(a). The fusion imaging results obtained by the FLBI algorithm, BOMP algorithm, and FLBIUB algorithm are shown in Figures 14(b)-14(d), respectively. It can be seen from Figure 14(b) that some false scatterers are introduced in the fusion imaging result obtained by the FLBI algorithm, which affects the recognition of the basic shape of the target. It can be seen from Figure 14(c) that the basic outline of the aircraft can be roughly distinguished from the fusion imaging result obtained by the BOMP algorithm. However, some scatterers of the nose and wing parts are lost, which affects the imaging quality. It can be seen from Figure 14(d) that the overall shape and detailed structure information can be clearly distinguished in the fusion imaging result obtained by the FLBIUB algorithm, which has better quality than the other two algorithms.

Case 2. Let $M_{1}=M_{2}=40$ and $N_{1}=N_{2}=40$; the two-dimensional observation echo data is shown in Figure 15(a). The fusion imaging results obtained by the FLBI algorithm, BOMP algorithm, and FLBIUB algorithm are shown in Figures 15(b)-15(d), respectively. It can be seen from Figure 15(b) that some structure information of the target is missing, and the complete shape and structure of the target cannot be reflected from the fusion imaging results obtained by the FLBI algorithm due to the reduction of the effective observation data. It can be seen from Figure 15(c) that some scatterers are missing and some false scatterers are introduced in the fusion imaging result obtained by the BOMP algorithm. It is difficult to distinguish the basic outline of the target. It can be seen from Figure 15(d) that a clear and complete target image can still be obtained by the FLBIUB algorithm with the reduction of the observation echo data.

Case 3. Let $M_{1}=M_{2}=30$ and $N_{1}=N_{2}=30$; the two-dimensional observation echo data is shown in Figure 16(a). The fusion imaging results obtained by the FLBI algorithm, BOMP algorithm, and FLBIUB algorithm are shown in Figures 16(b)-16(d), respectively. It can be seen from Figure 16(b) that the imaging resolution is low and the basic shape of the target cannot be judged from the fusion imaging result obtained by the FLBI algorithm due to the further reduction of the observation data. It can be seen from Figure 16(c) that a large number of false scatterers are introduced in the fusion imaging result obtained by the BOMP algorithm due to the limited observation echo data. The fusion image is seriously defocused, and it is impossible to distinguish the basic outline of the target. It can be seen from Figure 16(d) that a few scatterers in the nose part of the target are missing in the fusion imaging result obtained by the FLBIUB algorithm due to the limited observation echo data. However, a clear focused fusion image can still be obtained, and the basic outline of the target can be also distinguished from the result.

To further compare the quality of fusion imaging results obtained by the respective algorithms with different observation cases, image contrast (IC), target-to-background ratio (TBR) [26], and running time are utilized as the metrics. IC can evaluate the focus degree of an image. The higher the value, the more focused the image. TBR can effectively evaluate the noise suppression and focus degree of the image. The higher the value, the better denoising performance. Running time can reflect the calculation efficiency of the algorithm. The metrics of the fusion imaging results with different observation cases are shown in Table 2 . It can be seen that the IC and TBR of the imaging result obtained by the FLBIUB algorithm are both higher than those of the FLBI algorithm and BOMP algorithm in the same observation case. The running time of the FLBIUB algorithm is slightly longer than the FLBI algorithm but much shorter than the BOMP algorithm. It indicates that better fusion imaging quality can be obtained by the FLBIUB algorithm with fast imaging efficiency in two-dimensional ISAR fusion imaging of block structure targets.

4.2.2. Different SNRs. To analyze the noise influence for the proposed algorithm, the SNR of the observation echo data is changed to verify the antinoise performance with different SNRs. Let $M_{1}=M_{2}=50$ and $N_{1}=N_{2}=50$; FLBI algorithm, BOMP algorithm, and FLBIUB algorithm are utilized to achieve the multiradar fusion imaging when SNR is $20 \mathrm{~dB}$, $10 \mathrm{~dB}$, and $0 \mathrm{~dB}$. The fusion imaging results of the respective algorithms under different SNRs are shown in Figures 17-19, respectively. It can be seen from the imaging results that some noise is not suppressed, and some false scatterers are in the fusion imaging results obtained by the FLBI algorithm and BOMP algorithm as the SNR decreases. In particular, when SNR is $0 \mathrm{~dB}$, false scatterers increase apparently in the fusion imaging results obtained by the FLBI algorithm and BOMP algorithm, which seriously affects the imaging quality and makes it difficult to distinguish the basic shape and structure of the target. In contrast, the fusion imaging results obtained by the FLBIUB algorithm are clearer as the SNR decreases. Even when SNR is $0 \mathrm{~dB}$, a clear and clean fusion imaging result can still be obtained by the FLBIUB algorithm to distinguish the geometric structure of the target. It indicates that the FLBIUB algorithm has better noise suppression performance.

IC, TBR, and running time of the fusion imaging results obtained by different algorithms under different SNRs are shown in Table 3 . It can be seen from Table 3 that the IC and TBR of the fusion imaging results obtained by the same algorithm under different SNRs decrease with the decrease of SNR. Specifically, the variation of the FLBIUB algorithm is smaller than that of the other two algorithms. It shows that the antinoise performance of the FLBIUB algorithm is more robust. In addition, the IC and TBR of the fusion imaging results obtained by the FLBIUB algorithm are higher than those of the other two algorithms with the same SNR. It indicates that the image quality of the fusion imaging results obtained by the 


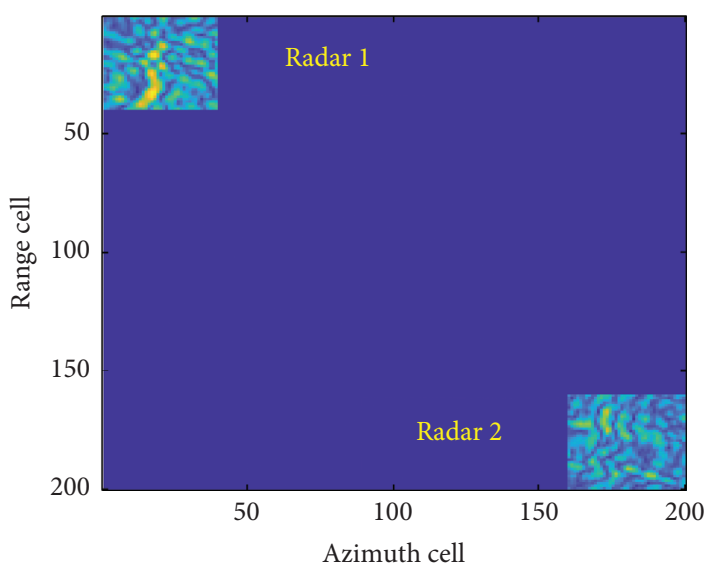

(a)

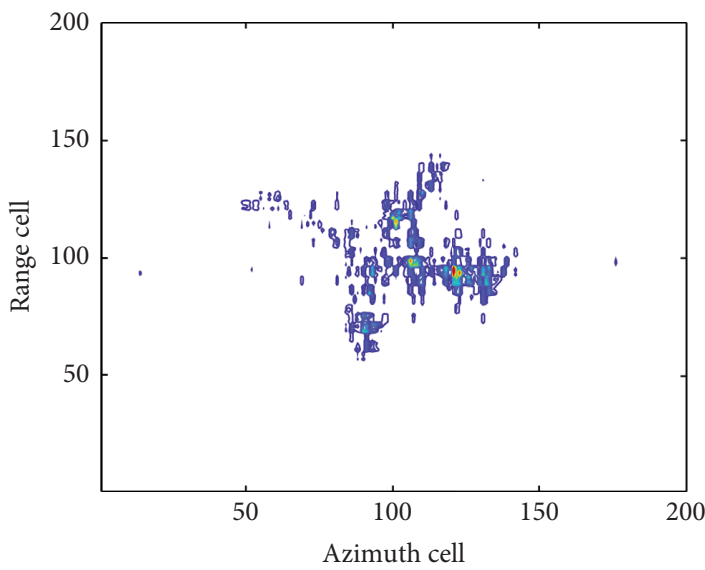

(c)

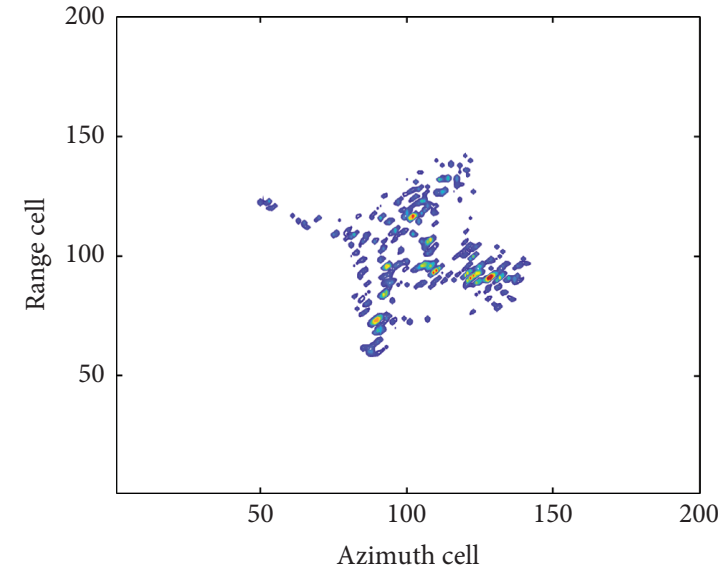

(b)

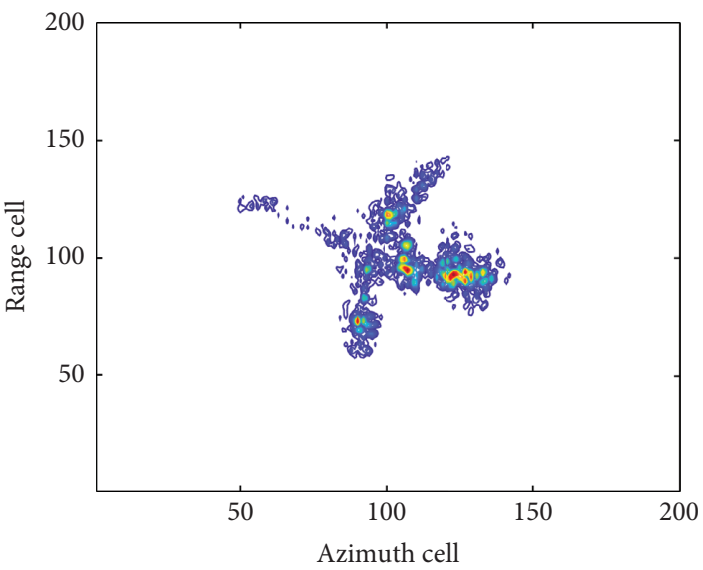

(d)

Figure 15: Two-dimensional observation echo data and fusion imaging results with $M_{1}=M_{2}=40$ and $N_{1}=N_{2}=40$. (a) Two-dimensional observation echo data. (b) Fusion imaging results of FLBI algorithm. (c) Fusion imaging results of BOMP algorithm. (d) Fusion imaging results of FLBIUB algorithm.

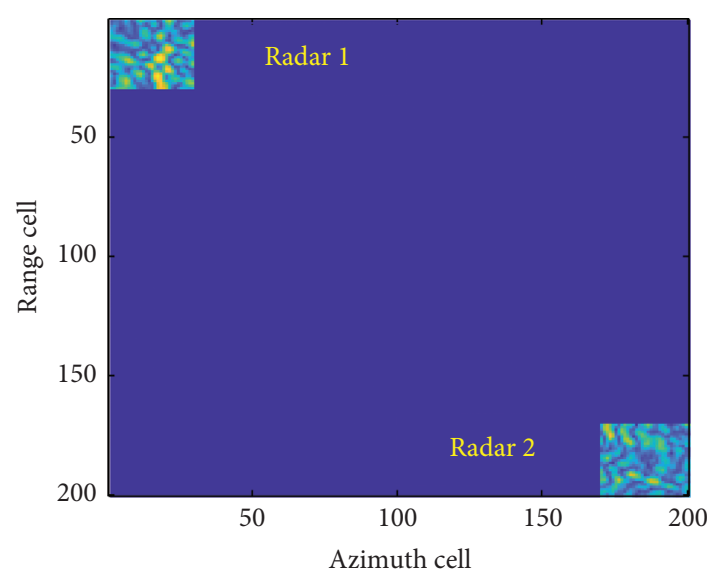

(a)

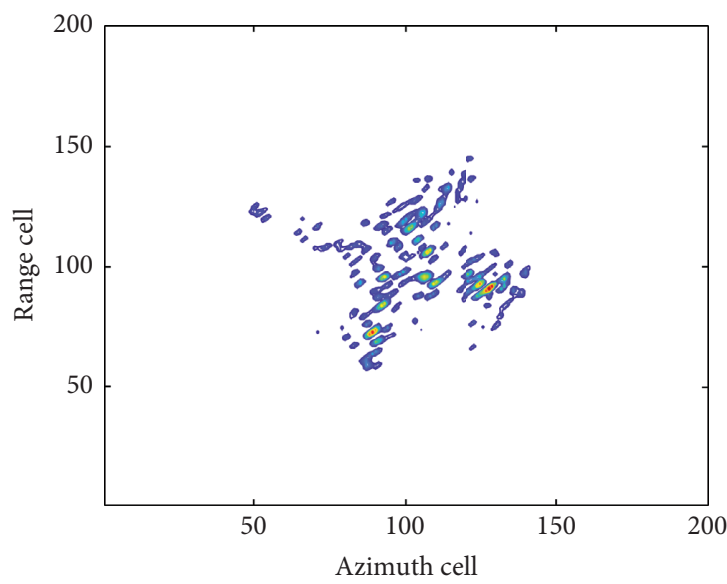

(b)

Figure 16: Continued. 


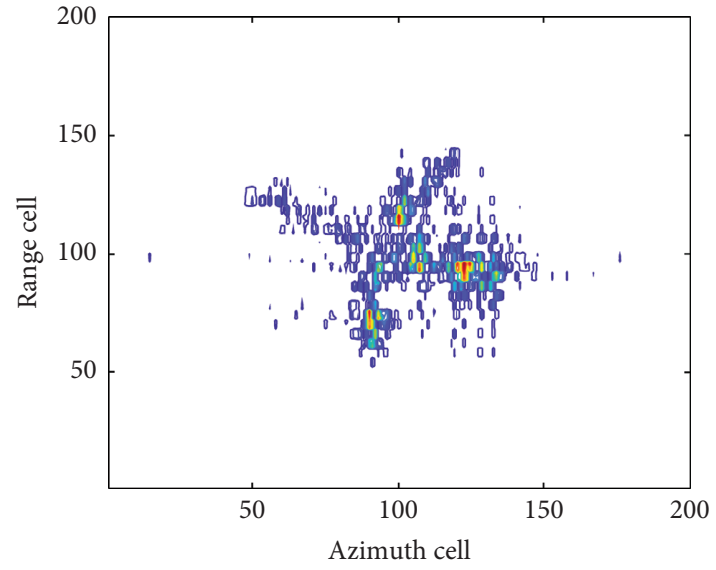

(c)

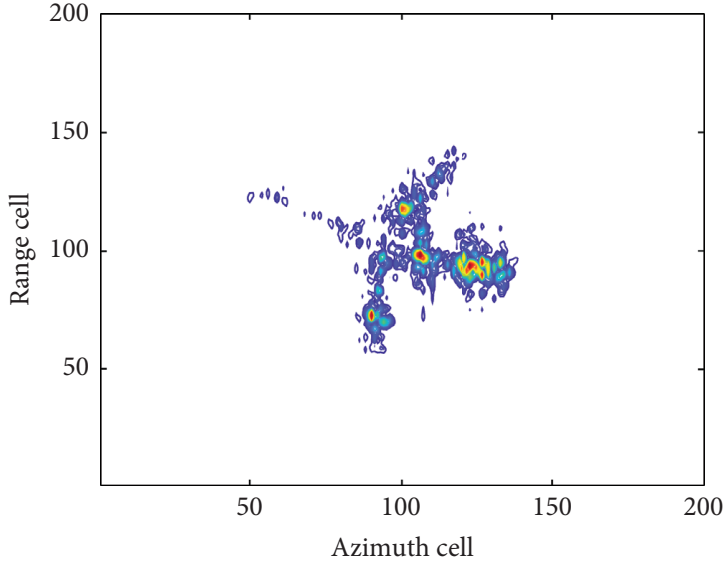

(d)

Figure 16: Two-dimensional observation echo data and fusion imaging results with $M_{1}=M_{2}=30$ and $N_{1}=N_{2}=30$. (a) Two-dimensional observation echo data. (b) Fusion imaging results of FLBI algorithm. (c) Fusion imaging results of BOMP algorithm. (d) Fusion imaging results of FLBIUB algorithm.

TABLE 2: The metrics of fusion imaging results with different observation cases.

\begin{tabular}{|c|c|c|c|c|}
\hline \multicolumn{2}{|c|}{ Case } & Case 1 & Case 2 & Case 3 \\
\hline \multirow{3}{*}{$I C$} & FLBI & 7.2041 & 6.4372 & 5.3421 \\
\hline & BOMP & 7.3429 & 6.4932 & 5.0953 \\
\hline & FLBIUB & 8.1294 & 7.8903 & 7.4945 \\
\hline \multirow{3}{*}{$T B R$} & FLBI & 28.8931 & 24.8492 & 21.9034 \\
\hline & BOMP & 29.3941 & 25.2703 & 20.0452 \\
\hline & FLBIUB & 37.4632 & 35.3942 & 31.9564 \\
\hline \multirow{3}{*}{ Running time (s) } & FLBI & 108.3 & 72.4 & 52.8 \\
\hline & BOMP & 384.4 & 255.9 & 173.4 \\
\hline & FLBIUB & 146.8 & 106.6 & 76.4 \\
\hline
\end{tabular}

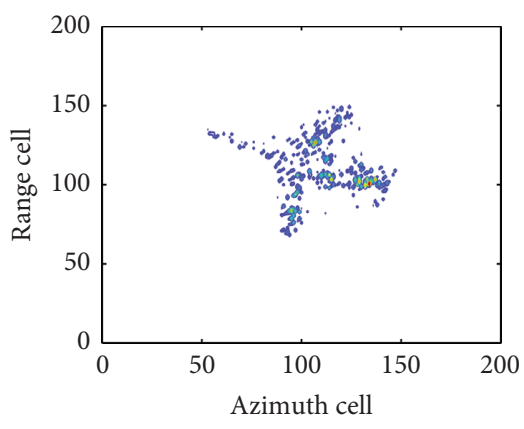

(a)

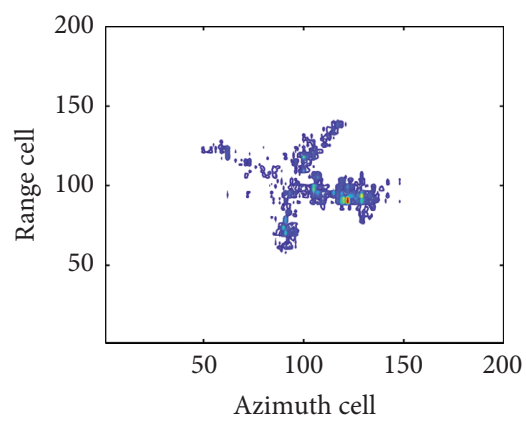

(b)

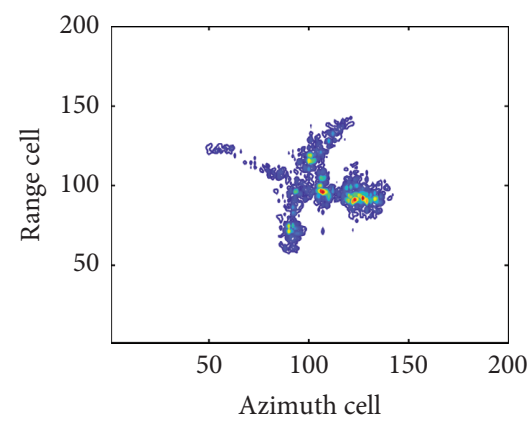

(c)

FIGURE 17: Fusion imaging results obtained by different algorithms when SNR is $20 \mathrm{~dB}$. (a) FLBI algorithm. (b) BOMP algorithm. (c) FLBIUB algorithm. 


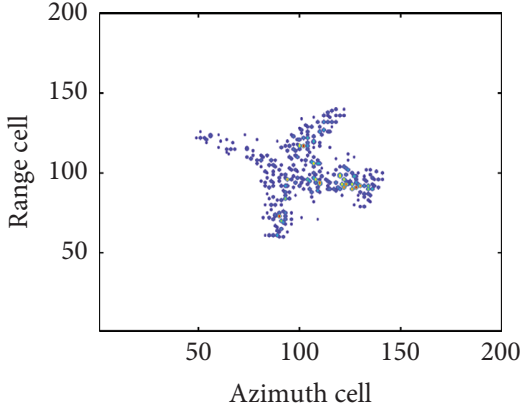

(a)

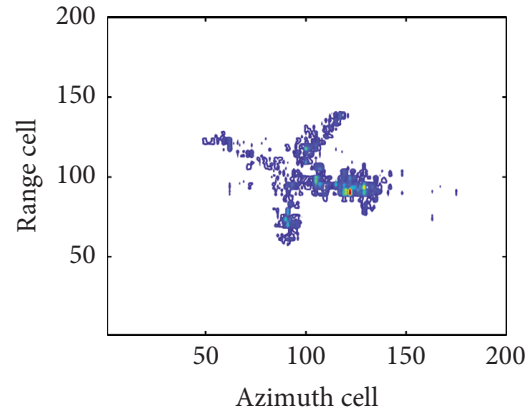

(b)

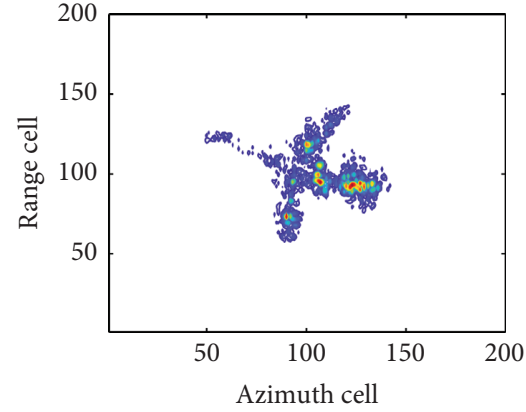

(c)

FIGURE 18: Fusion imaging results obtained by different algorithms when SNR is $10 \mathrm{~dB}$. (a) FLBI algorithm. (b) BOMP algorithm. (c) FLBIUB algorithm.

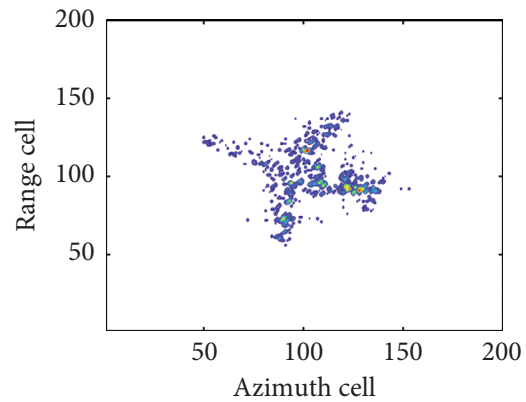

(a)

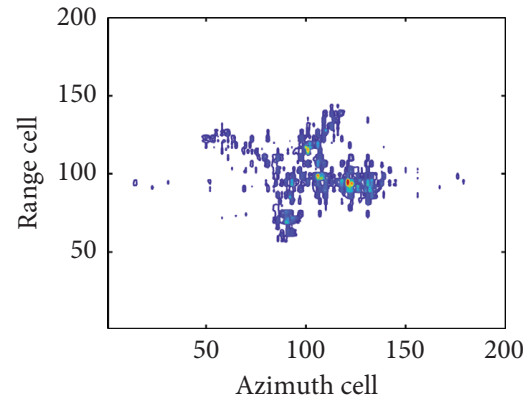

(b)

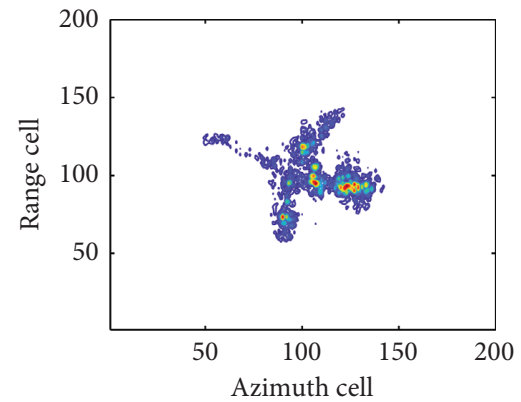

(c)

FIGURE 19: Fusion imaging results obtained by different algorithms when SNR is 0 dB. (a) FLBI algorithm. (b) BOMP algorithm. (c) FLBIUB algorithm.

TABle 3: The metrics of fusion imaging results under different SNRs.

\begin{tabular}{|c|c|c|c|c|}
\hline \multicolumn{2}{|c|}{$\mathrm{SNR} / \mathrm{dB}$} & 20 & 10 & 0 \\
\hline \multirow{3}{*}{ IC } & FLBI & 7.2041 & 6.7042 & 6.1131 \\
\hline & BOMP & 7.3429 & 6.6731 & 6.0324 \\
\hline & FLBIUB & 8.1294 & 7.6334 & 7.1935 \\
\hline \multirow{3}{*}{$T B R$} & FLBI & 28.8931 & 25.2423 & 21.0294 \\
\hline & BOMP & 29.3941 & 25.4561 & 20.2853 \\
\hline & FLBIUB & 37.4632 & 34.5819 & 31.6432 \\
\hline \multirow{3}{*}{ Running time (s) } & FLBI & 108.3 & 109.2 & 112.9 \\
\hline & BOMP & 384.4 & 386.3 & 385.3 \\
\hline & FLBIUB & 146.8 & 149.2 & 148.7 \\
\hline
\end{tabular}

FLBIUB algorithm is better than that of the other algorithms when achieving multiradar fusion imaging of block structure targets. Furthermore, the running time of the three algorithms is close with the same SNR and changes little with the variation of SNR. It shows that the running time of the algorithms changes little when SNR changes.

\section{Conclusions}

A two-dimensional multiradar ISAR fusion imaging method based on the FLBIUB algorithm is proposed to improve the two-dimensional resolution of ISAR imaging of block structure targets. A vectorized sparse representation model of multiradar ISAR fusion imaging is established based on the GTD model, which can characterize the scattering characteristics of the echo signal better than the ideal scatterer model. Considering the block sparsity of the echo data, the FLBIUB algorithm is proposed to solve the sparse representation problem on a large scale and achieve two-dimensional ISAR fusion imaging. The weighted back-adding residual and the condition number optimization of the basis matrix are combined to accelerate the iteration convergence speed 
and improve the efficiency of fusion imaging further. The experimental results verify the effectiveness and superiority of the proposed algorithm.

\section{Data Availability}

No data were used to support this study.

\section{Conflicts of Interest}

The authors declare that there are no conflicts of interest regarding the publication of this paper.

\section{Acknowledgments}

This work was supported by the National Natural Science Foundation of China, under Grant no. 61601496, the Natural Science Foundation of Hebei Province of China, under Grant nos. F2019506031, F2019506037, and F2020506036, the Frontier Innovation Program of Army Engineering University, under Grant no. KYSZJQZL2005, the Basic Frontier Science and Technology Innovation Program of Army Engineering University, under Grant no. KYSZJQZL2020, and the Research Innovation Development Funding of Army Engineering University Shijiazhuang Campus, under Grant no. KYSZJQZL1902.

\section{References}

[1] F. Zhou and X. Bai, "High-resolution sparse subband imaging based on Bayesian learning with hierarchical priors," IEEE Transactions on Geoscience and Remote Sensing, vol. 56, no. 8, pp. $4568-4580,2018$.

[2] X. He, N. Tong, and X. Hu, "High-resolution ISAR imaging via MMV-based block-sparse signal recovery," IET Radar, Sonar \& Navigation, vol. 13, no. 2, pp. 208-212, 2019.

[3] L. Sun and W. Chen, "Improved bayesian ISAR imaging by learning the local structures of the target scene," IEEE Sensors Journal, vol. 19, no. 19, pp. 8865-8877, 2019.

[4] S. Wei, L. Zhang, H. Ma, and H. Liu, "Sparse frequency waveform optimization for high-resolution ISAR imaging," IEEE Transactions on Geoscience and Remote Sensing, vol. 58, no. 1 , pp. 546-566, 2020.

[5] Y. Zhang, T. Wang, H. Zhao, Y. Zhang, and H. Zhao, "Multiple radar subbands fusion algorithm based on support vector regression in complex noise environment," IEEE Transactions on Antennas and Propagation, vol. 66, no. 1, pp. 381-392, 2018.

[6] J. Rong, Y. Wang, and T. Han, "Iterative optimization-based ISAR imaging with sparse aperture and its application in interferometric ISAR imaging," IEEE Sensors Journal, vol. 19, no. 19, pp. 8681-8693, 2019.

[7] F. Ye, D. Liang, and J. Zhu, "ISAR enhancement technology based on compressed sensing," Electronics Letters, vol. 47, no. 10 , pp. $620-621,2011$.

[8] D. L. Donoho, "Compressed sensing," IEEE Transactions on Information Theory, vol. 52, no. 4, pp. 1289-1306, 2006.

[9] Z. Yin, X. Lu, and W. Chen, "Echo preprocessing to enhance SNR for 2D CS-based ISAR imaging method," Sensors, vol. 18, no. 12, p. 4409, 2018.
[10] L. Wang, L. Zhao, G. Bi, C. Wan, and L. Yang, "Enhanced ISAR imaging by exploiting the continuity of the target scene," IEEE Transactions on Geoscience and Remote Sensing, vol. 52, no. 9, pp. 5736-5750, 2014.

[11] Y. C. Eldar, P. Kuppinger, and H. Bolcskei, "Block-sparse signals: uncertainty relations and efficient recovery," IEEE Transactions on Signal Processing, vol. 58, no. 6, pp. 30423054, 2010.

[12] Y. C. Eldar and M. Mishali, "Robust recovery of signals from a structured union of subspaces," IEEE Transactions on Information Theory, vol. 55, no. 11, pp. 5302-5316, 2009.

[13] Z. Zhang and B. D. Rao, "Sparse signal recovery with temporally correlated source vectors using sparse Bayesian learning," IEEE Journal of Selected Topics in Signal Processing, vol. 5, no. 5, pp. 912-926, 2011.

[14] J. Fang, Y. Shen, H. Li, and P. Wang, "Pattern-coupled sparse bayesian learning for recovery of block-sparse signals," IEEE Transactions on Signal Processing, vol. 63, no. 2, pp. 360-372, 2015.

[15] W. Yin, S. Osher, D. Goldfarb, and J. Darbon, "Bregman iterative algorithms for \$lell_1\$-Minimization with applications to compressed sensing," SIAM Journal on Imaging Sciences, vol. 1, no. 1, pp. 143-168, 2008.

[16] W. Yin, "Analysis and generalizations of the linearized bregman method," SIAM Journal on Imaging Sciences, vol. 3, no. 4, pp. 856-877, 2010.

[17] S. Osher, Y. Mao, B. Dong et al., "Fast linearized bregman iteration for compressive sensing and sparse denoising," Communications in Mathematical Sciences, vol. 8, no. 1, pp. 93-111, 2011.

[18] S. Li, W. Chen, X. Ma, and J. Yang, "A fast complex linearized Bregman iteration algorithm and its application in ISAR imaging," Scientia Sinica Informationis, vol. 45, no. 9, pp. 1179-1196, 2015.

[19] L. Xiang, S. Li, J. Yang, W. Chen, and H. Xiang, "A Fast decoupled ISAR high-resolution imaging method using structural sparse information under low SNR," Journal of Systems Engineering and Electronics, vol. 30, no. 3, pp. 492503, 2019.

[20] B. Tian, Z. Chen, and S. Xu, "Sparse subband fusion imaging based on parameter estimation of geometrical theory of diffraction model," IET Radar, Sonar \& Navigation, vol. 8, no. 4, pp. 318-326, 2014.

[21] S. Li, W. Chen, W. Liu, J. Yang, and X. Ma, "Fast 2D super resolution ISAR imaging method under low signal-to-noise ratio," IET Radar, Sonar \& Navigation, vol. 11, no. 10, pp. 1495-1504, 2017.

[22] S. Li, G. Zhao, W. Zhang, Q. Qiu, and H. Sun, "ISAR imaging by two-dimensional convex optimization-based compressive sensing," IEEE Sensors Journal, vol. 16, no. 19, pp. 7088-7093, 2016.

[23] W. Deng, W. Yin, and Y. Zhang, Group Sparse Optimization by Alternating Direction Method, Rice University, Houston, TX, USA, 2011.

[24] J.-F. Cai, S. Osher, and Z. Shen, "Linearized bregman iterations for frame-based image deblurring," SIAM Journal on Imaging Sciences, vol. 2, no. 1, pp. 226-252, 2009.

[25] J. A. Tropp and A. C. Gilbert, "Signal recovery from random measurements via orthogonal matching pursuit," IEEE Transactions on Information Theory, vol. 53, no. 12, pp. 4655-4666, 2007.

[26] X. Zhu, B. Guo, W. Hu, L. Shi, J. Ma, and D. Xue, "Scene segmentation of multi-band ISAR fusion imaging based on MB-PCSBL," IEEE Sensors Journal, vol. 21, no. 3, pp. 3520-3532, 2021. 